\title{
Schlafen 4-expressing myeloid-derived suppressor cells are induced during murine gastric metaplasia
}

\author{
Lin Ding, ${ }^{1}$ Michael M. Hayes, ${ }^{1}$ Amanda Photenhauer, ${ }^{1}$ Kathryn A. Eaton,, ${ }^{2}$ Qian Li, ${ }^{1}$ Ramon Ocadiz-Ruiz, ${ }^{1}$ and Juanita L. Merchant ${ }^{1,3}$ \\ 'Department of Internal Medicine-Castroenterology, ${ }^{2}$ Unit for Laboratory Animal Medicine, and ${ }^{3}$ Department of Molecular and Integrative Physiology, University of Michigan, Ann Arbor, Michigan, USA.
}

\begin{abstract}
Chronic Helicobacter pylori infection triggers neoplastic transformation of the gastric mucosa in a small subset of patients, but the risk factors that induce progression to gastric metaplasia have not been identified. Prior to cancer development, the oxyntic gastric glands atrophy and are replaced by metaplastic cells in response to chronic gastritis. Previously, we identified schlafen 4 (SIfn4) as a GLI1 target gene and myeloid differentiation factor that correlates with spasmolytic polypeptide-expressing metaplasia (SPEM) in mice. Here, we tested the hypothesis that migration of SLFN4-expressing cells from the bone marrow to peripheral organs predicts preneoplastic changes in the gastric microenvironment. Lineage tracing in Helicobacter-infected SIfn4 reporter mice revealed that SLFN4+ cells migrated to the stomach, where they exhibited myeloid-derived suppressor cell (MDSC) markers and acquired the ability to inhibit T cell proliferation. SLFN4 ${ }^{+}$ MDSCs were not observed in infected CLI1-deficient mice. Overexpression of sonic hedgehog ligand (SHH) in infected WT mice accelerated the appearance of SLFN4+ MDSCs in the gastric corpus. Similarly, in the stomachs of $H$. pylori-infected patients, the human SLFN4 ortholog SLFN12L colocalized to cells that expressed MDSC surface markers CD15 ${ }^{+}$CD $33^{+}+\mathrm{HLA}-$ $D^{10}$. Together, these results indicate that SLFN4 marks a GLI1-dependent population of MDSCs that predict a shift in the gastric mucosa to a metaplastic phenotype.
\end{abstract}

\section{Introduction}

Helicobacter pylori infection induces chronic inflammation in the stomach, which eventually leads to atrophy of the acid-producing glands, metaplasia, dysplasia, and then gastric cancer in some infected patients (1-3). The timeline for gastric cancer development occurs over several decades, raising the likelihood that Helicobacter infection is only one of several factors contributing to transformation of the normal gastric epithelium. Indeed, results from The Cancer Genome Atlas (TCGA) for gastric cancer reported only sporadic molecular signatures of Helicobacter in primary tumors (4). Therefore, infection with Helicobacter alone is not sufficient to predict who among the small subset of infected patients will develop gastric cancer. In most instances, the extent of the inflammatory response also contributes to cancer susceptibility $(5,6)$. Nevertheless, at least $50 \%$ of infected individuals develop chronic gastritis without symptoms or further progression of the mucosa to metaplasia and dysplasia (7). Both intestinal metaplasia and spasmolytic polypeptide-expressing metaplasia (SPEM) are histologic lesions strongly associated with neoplastic transformation (8-11). Identifying markers predictive of preneoplasia would allow clinicians to risk-stratify the subset of individuals at greater risk for progression to gastric cancer and who subsequently require more frequent monitoring, regardless of Helicobacter prevalence (12).

Hedgehog $(\mathrm{HH})$ ligands expressed in the gastrointestinal epithelium activate GLI transcription factors in stromal and immune cells (13). Zavros and coworkers showed that the sonic hedgehog (SHH) ligand secreted from gastric parietal cells within 2 days after the infection is required for myeloid cell recruitment to the infect-

Conflict of interest: The authors have declared that no conflict of interest exists. Submitted: April 30, 2015; Accepted: May 19, 2016.

Reference information: / Clin Invest. 2016;126(8):2867-2880. doi:10.1172/JCI82529. ed mouse stomach (14). After 2 months of Helicobacter infection, $\mathrm{SHH}$ expression in parietal cells gradually diminishes in response to chronic gastritis, despite adjacent mucous cells retaining ligand expression (15). Moreover, GLI1 ${ }^{+}$myeloid cells are recruited to the stomach within the initial months of the infection (16). By 6 months, most of the parietal and zymogenic chief cells have atrophied and are replaced by SPEM. However, deletion of one or both Gli1 alleles prevents Helicobacter-induced SPEM, indicating that targets of the HH pathway are required for SPEM development (16). Since HH signaling in the stomach is paracrine (17), blocking $\mathrm{HH}$-dependent crosstalk between infiltrating immune cells and the gastric epithelium is sufficient to prevent histologic transition from chronic gastritis to metaplasia.

A myeloid cell differentiation factor and GLI1 target gene (18) called schlafen 4 (Slfn4) was identified in a microarray analysis of gastric tissue from Helicobacter-infected WT versus $\mathrm{Gli1}^{-/-}$mice (16). SLFN $4^{+}$myeloid cells express IL-1 $\beta$ and TNF- $\alpha$ (16), cytokines associated with myeloid-derived suppressor cell (MDSC) regulation $(6,19)$. MDSCs are a heterogeneous myeloid cell population that develops under conditions of infection, tissue injury, autoimmune disease, and cancer (20). Their ability to suppress $\mathrm{T}$ cell function dampens the immune response and creates a microenvironment favoring neoplastic transformation (21).

Here we tested the hypothesis that Slfn 4 expression marks a GLI1-dependent population of myeloid cells with phenotypic characteristics of MDSCs. We found that SLFN4 ${ }^{+}$cells in the mouse stomach coincided with SPEM in the setting of chronic gastritis before the development of dysplasia. Since type I interferon induction of Slfn 4 gene expression required GLI1, we concluded that $\mathrm{HH}$ signaling synergizes with regulatory cytokines to create a permissive environment for gastric metaplasia, a harbinger of possible neoplastic transformation. 

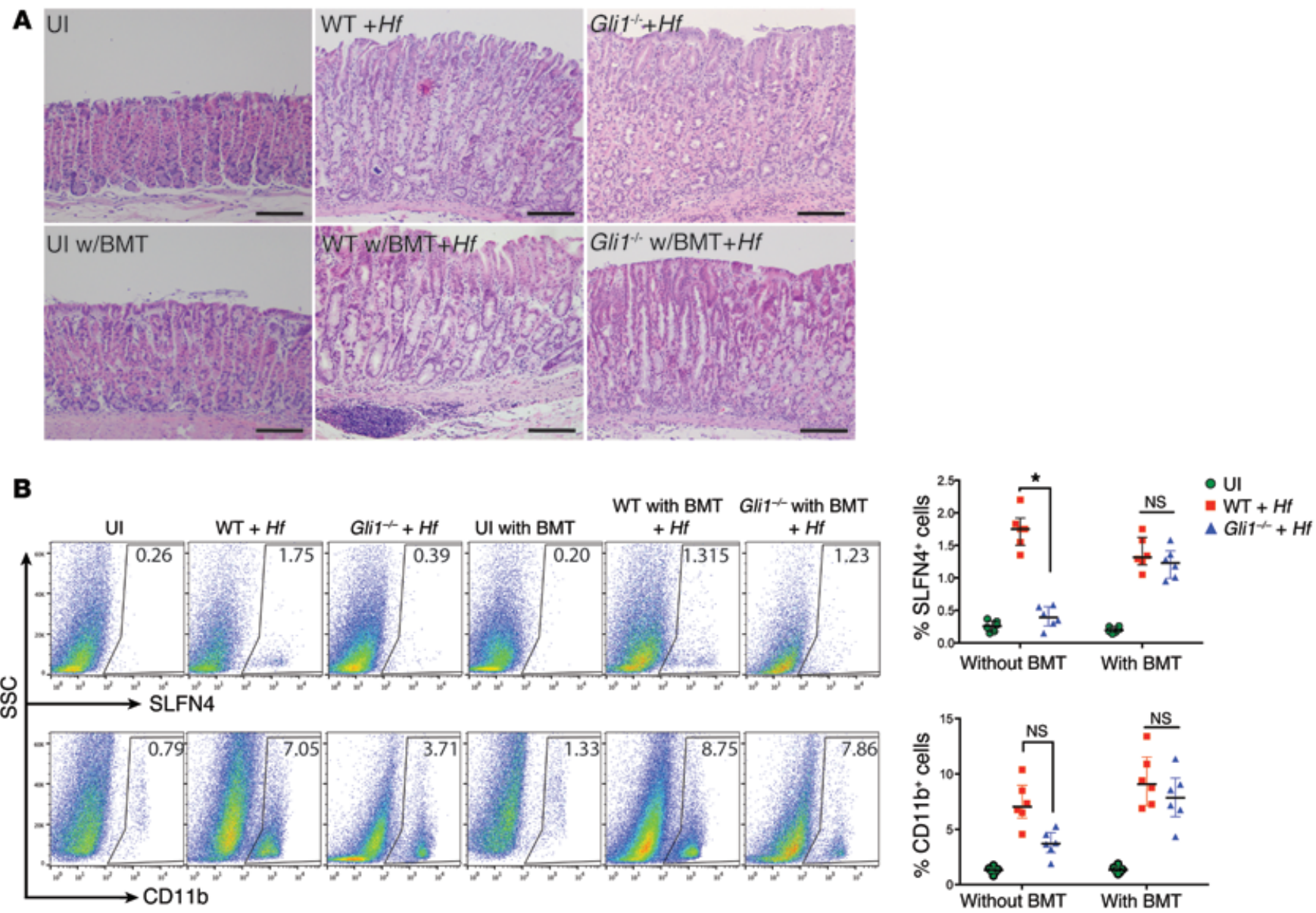

Figure 1. Bone marrow-derived cells are sufficient to induce SPEM with $\boldsymbol{H}$. felis. (A) Representative H\&E images $(n=6)$ for each group as indicated with and without bone marrow transplant (BMT) (from SIfn4-CreER ${ }^{T 2}$ Rosa26-LSL-tdTomato [SIfn4-tdT] mice) with and without $H$. felis infection (Hf). UI, uninfected. Scale bars: $50 \mu \mathrm{m}$. (B) Flow analysis of SLFN4-tdT ${ }^{+}$or CD11b+ cells versus side scatter (SSC). The percentage of SLFN4 ${ }^{+}$or CD11 b+ cells per mouse is shown in the representative histograms and plotted in the adjacent scatter graphs for $n=6$ mice per group over 3 experiments. Horizontal lines represent the median and interquartile range. Significance was determined using Kruskal-Wallis ANOVA with Dunn's test of multiple comparisons. ${ }^{*} P<0.05$.

\section{Results}

SPEM development requires Hedgehog signaling. Helicobacterinduced SPEM in WT mice was observed at 6 months after infection. Infected $\mathrm{Gli}^{\mathrm{nLacZ} / \mathrm{nLacZ}}$ mice (referred to here as $\mathrm{Gli1}^{1^{-/}}$mice) did not develop SPEM as previously reported, which implicates a significant role for canonical $\mathrm{HH}$ signaling in the emergence of this preneoplastic lesion (Figure 1A and ref. 16). In the stomach, stromal cells, specifically myofibroblasts and immune cells, express GLI1 $(16,17,22)$. To determine whether the metaplastic change was due to bone marrow-derived cells (BMDCs), we transferred marrow from Slfn4-eGFP-CreER ${ }^{T 2}$ Rosa26-LSL-tdTomato (Slfn4$t d T$; see below) mice (that are $\mathrm{Gli1}^{+/+}$) into irradiated WT (WT w/bone marrow transplant [BMT]) or $\mathrm{Gli1}^{-/-}$mice $\left(\mathrm{Gli1}^{-/-} \mathrm{w} / \mathrm{BMT}\right)$ prior to infection with $\mathrm{H}$. felis for 6 months. Infected $\mathrm{Gli1}^{-/-}$w/BMT mice that received WT marrow developed SPEM, demonstrating that BMDCs were sufficient to transfer gastric susceptibility to Helicobacter-induced metaplasia (Figure 1A). SPEM is defined by the combined expression of chief cell (e.g., gastric intrinsic factor) and mucous neck cell (e.g., TFF2 and GSII-lectin) markers that represent reprogramming of the mature gastric chief cells in the setting of parietal cell atrophy (23-25). Overlap between these two cell-specific markers confirmed the presence of SPEM in the infected mice with WT marrow (Supplemental Figure 1; supplemental material available online with this article; doi:10.1172/ JCI82529DS1). WT mice developed metaplastic changes only if infected, and the SPEM correlated with an increase in CD11b ${ }^{+}$and SLFN $4^{+}$cells (Figure 1B). However, the absence of SPEM in the $\mathrm{Gli1}^{-/-}$mice correlated with reduced numbers of SLFN4 ${ }^{+}$, but not a significant reduction of $\mathrm{CD}^{11 \mathrm{~b}^{+}}$cells (Figure 1B), suggesting a shift in the composition of the myeloid population rather than a defect in total myeloid cell recruitment.

SHH accelerates $H$. felis-induced gastric metaplasia. Although these results collectively indicated that $\mathrm{HH}$ signaling was necessary for SPEM development, the phenotype required several months to develop. To accelerate the timeline, we infected transgenic mice expressing the $p C M V$-Shh transgene (16) with $H$. felis over 6 months and compared them with infected nontransgenic mice. Serum SHH levels in uninfected $p C M V$-Shh transgenic mice were 4 times the levels observed in WT mice (Figure 2A). In addition, there were more immune cells observed in the $p C M V$ $S h h$ transgenic mice in the absence of Helicobacter infection compared with WT mice (Supplemental Figure 2). With Helicobacter 
A

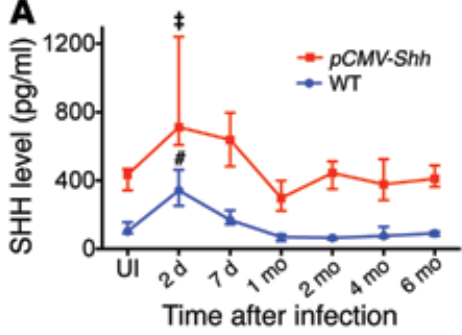

C

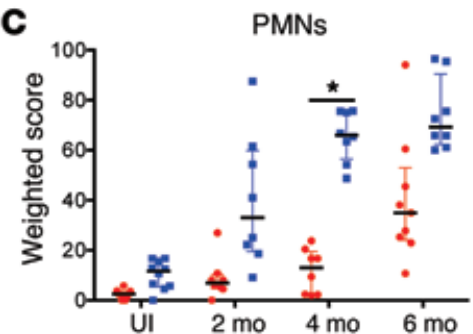

D

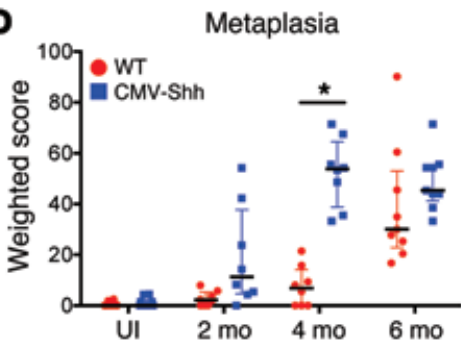

E

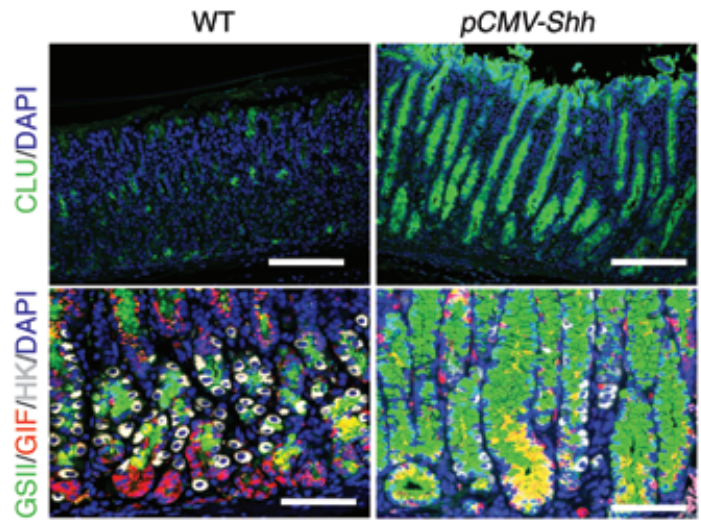

infection, there was a rapid increase in the blood levels of $\mathrm{SHH}$, which peaked within days after the infection and then returned to baseline levels after 1 month (Figure 2A). The result was consistent with a prior report indicating that $H$. pylori infection increases circulating levels of $\mathrm{SHH}$, which originates from gastric parietal cells (14). H. felis-infected $p C M V$-Shh mice exhibited greater inflammation in the gastric corpus at 2 months, and subsequently SPEM was observed at 4 months, which was 2 months earlier than observed in WT littermates (Figure 2B). The amount of polymorphonuclear (PMN) leukocytes infiltrating the mucosa and the extent of SPEM, as quantified by morphometry, were greater in the SHH-overexpressing mice (Figure 2, C and D). SPEM development in $p C M V$ Shh mice was also documented by de novo expression of clusterin, as well as coexpression of GSII lectin and GIF at the oxyntic gland base, with atrophy of $\mathrm{H}^{+}-\mathrm{K}^{+}$-ATPase-positive parietal cells
(Figure 2E). Real-time quantitative PCR (RT-qPCR) analysis for $\mathrm{H}^{+}-\mathrm{K}^{+}$-ATPase $\alpha$ subunit (Atp4a), Gif, mucous neck cell and SPEM cell marker trefoil factor 2 (Tff2), and clusterin mRNAs were used to quantify the extent of the metaplastic epithelial response (Supplemental Figure 3). Unlike the corpus, the antrum exhibited no phenotypic changes (Supplemental Figure 4).

SHH accelerates the appearance of SLFN4. Since ectopic expression of SHH accelerated SPEM development, we queried whether the appearance of SLFN4 ${ }^{+}$cells also occurred earlier. A Slfn4eGFP-CreER ${ }^{T 2}$ BAC transgene was generated to trace SLFN4 ${ }^{+}$ cells from the bone marrow by breeding this transgenic line to Rosa26-LSL-tdTomato mice to produce the transgenic line Slfn4eGFP-CreER ${ }^{T 2}$ Rosa26-LSL-tdTomato (referred to here as Slfn4$t d T$ mice), which served as bone marrow donors (Figure $3 \mathrm{~A}$ ). Bone marrow cells from the Slfn4-tdT mice were injected into 8-week- 
A

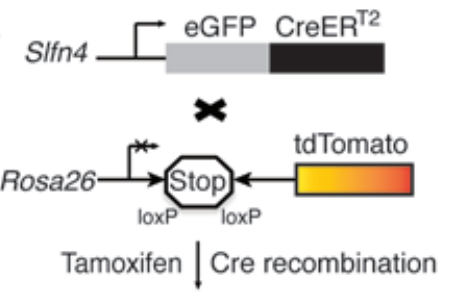

Rosa26 $\longrightarrow$ tdTomato

B

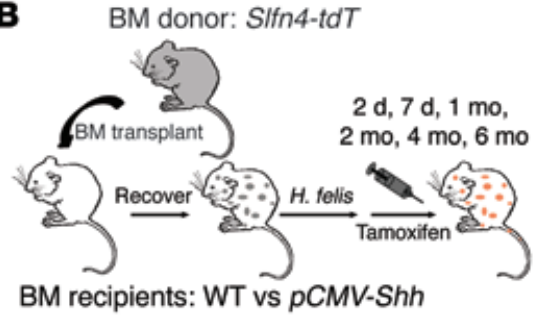

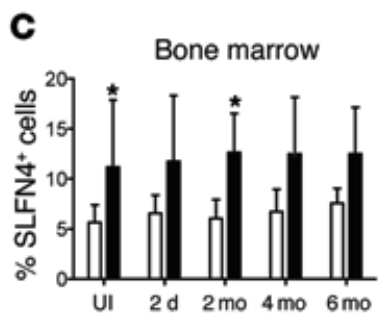

D

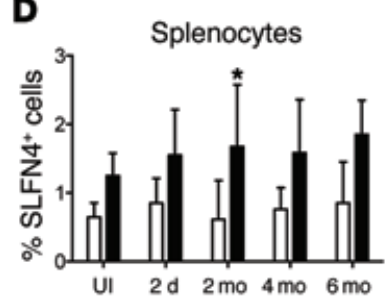

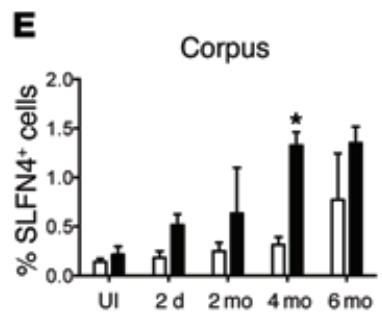

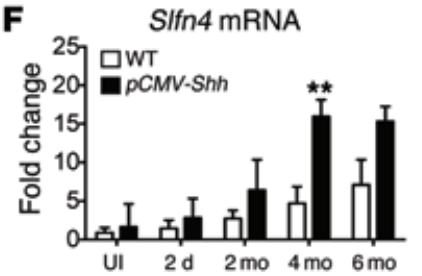

G

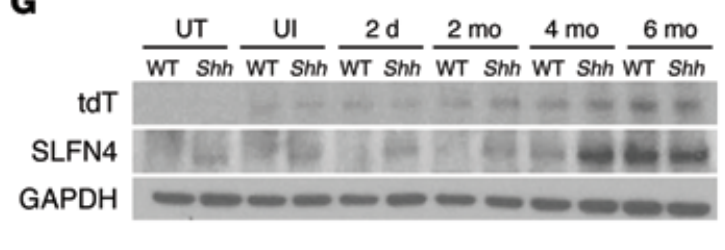

H

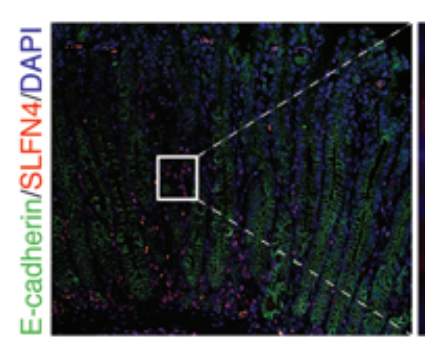

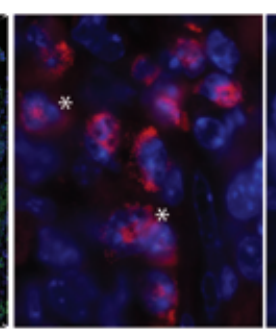

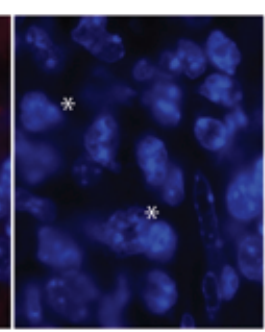

I

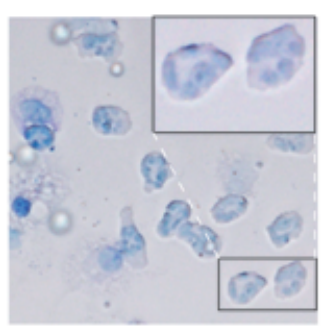

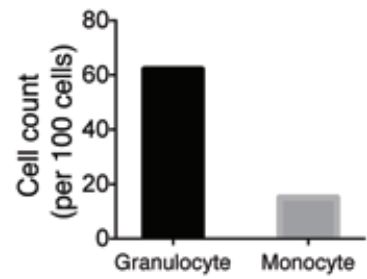

Figure 3. SLFN4+ cells track to the stomach after $\boldsymbol{H}$. felis infection. (A) Schematic diagram of SIfn4-tdT (SIfn4-CreER ${ }^{T 2}$ Rosa26-LSL-tdTomato) mice generated. (B) Protocol to lineage trace SLFN4-expressing cells from the bone marrow to peripheral organs. WT versus pCMV-Shh chimeric mice reconstituted with SIfn4-tdT bone marrow were treated with Tx 2 weeks prior to euthanization. Shown is the percentage of SLFN4+ cells in the (C) bone marrow, (D) spleen, and (E) corpus by flow cytometry, plotted as the median and interquartile range for $n=8-10$ mice per group over 3 experiments. Kruskal-Wallis ANOVA with Dunn's test of multiple comparisons was performed for C-E; ${ }^{*} P<0.05$. The corpus was analyzed for (F) SIfn4 mRNA by RT-qPCR and (G) tdT, SLFN4, and GAPDH protein by Western blot for $n=8-10$ mice. One-way ANOVA followed by Tukey's multiple comparisons test on log-transformed RT-qPCR values was performed for $\mathbf{F}$; ${ }^{* *} P<0.01$. UT, untreated. (H) Representative images from $n=5 p C M V$-Shh mouse corpora infected for 4 months showing SLFN4-tdT red ${ }^{+}$cells with E-cadherin (green) and DAPI. Left: original magnification, $\times 200$. White box region enlarged in the middle (tdT and DAPI) and right (DAPI only) panels (magnification, $\times 1,000$ ). Asterisks indicate granulocytic nuclear morphology. (I) Giemsa stain of SLFN4-tdT+ cells sorted from $n=3$ infected $p C M V$-Shh mouse stomachs (original magnification, $\times 600$ ) showing granulocytic and monocytic cells enumerated per 100 cells. Black box indicates enlarged region (original magnification, $\times 1,000$ ).

old irradiated WT and $p C M V$-Shh mice. After a 6-week engraftment period, the two groups were infected with $H$. felis, then pulsed with tamoxifen ( $\mathrm{Tx}, 0.1 \mathrm{mg} / \mathrm{g}$ body weight/d) 2 weeks before euthanization at the indicated time points (Figure 3B). The percentage of SLFN $4^{+}$cells from the bone marrow, spleen, liver, intestine, and stomach corpus was analyzed. When we compared the uninfected $p C M V$-Shh mice with WT mice, there was a notable increase in the percentage of SLFN $4^{+}$cells in the bone marrow and spleen, with a pronounced trend toward significance (Supplemental Figure 5A), but no differences were observed in the corpus (stomach), liver, or intestine in the absence of the Helicobacter infection (Supplemental Figure 5, A, C, and D). After infection with H. felis, the number of SLFN4-expressing cells remained similar in the bone marrow and spleen of the $p C M V$-Shh mice compared with WT mice (Figure 3, C and D, and Supplemental Figure 5B). Moreover, there was no significant increase in the number of SLFN $4^{+}$cells in the liver and intestine with infection over 6 months (Supplemental Figure $5, \mathrm{C}$ and D). However, in the gastric corpus, there was a time- dependent increase in SLFN4 $4^{+}$cells that correlated with the duration of Helicobacter infection (Figure 3E and Supplemental Figure 5B). Slfn 4 mRNA and SLFN4 protein were detectable in total cell lysates from the corpus, and expression peaked at 4 and 6 months after $\mathrm{H}$. felis infection with the appearance of SPEM in the $p C M V$ Shh and WT mice, respectively (Figure $3 \mathrm{~F}$ and Figure 2B). Using gastric lysates, a Western blot analysis of the tdTomato (tdT) protein (red fluorescent protein) matched the pattern of endogenous SLFN4 expression, except in mice without BMT (untreated [UT]), which did not express tdT. Over the subsequent months after infection, both endogenous SLFN4 protein and the tdT protein were at low levels until 4 months after the infection, when Slfn 4 expression peaked in the $p C M V$-Shh mice (Figure 3G). Epifluorescence revealed the presence of SLFN $4^{+}$cells in the lamina propria of the infected $p C M V$-Shh corpus 4 months after infection (Figure $3 \mathrm{H}$ ). A magnified view of the $\mathrm{tdT}^{+}$cells in the lamina propria showed the presence of granulocytic nuclei in the SLFN4-tdT ${ }^{+}$cells. To further identify the nuclear morphology of the SLFN $4^{+}$cells from 
A

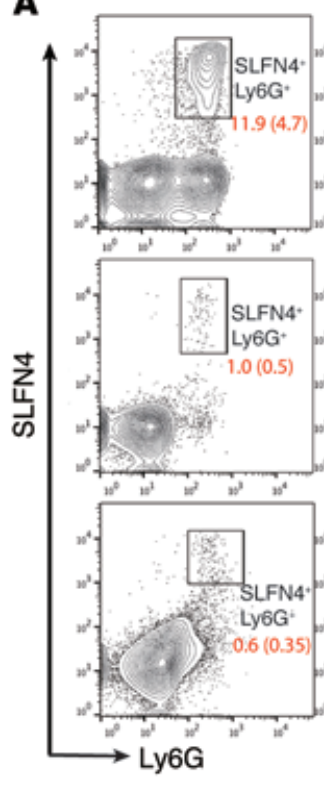

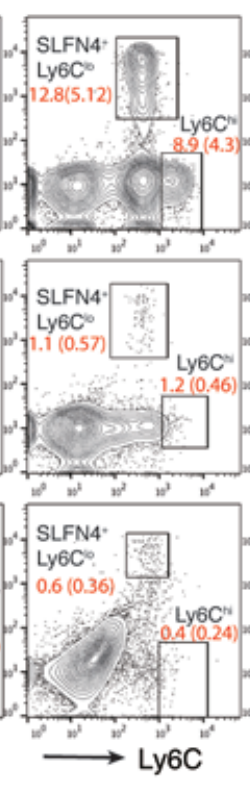

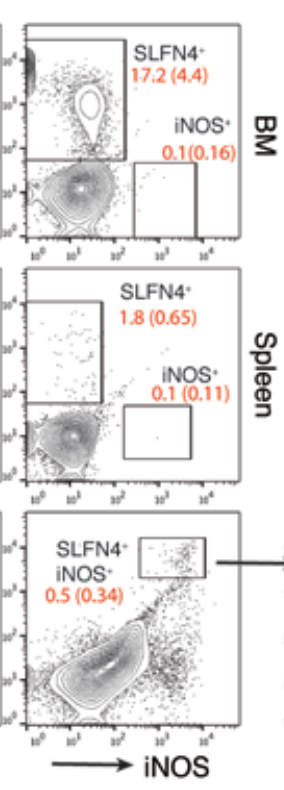

B

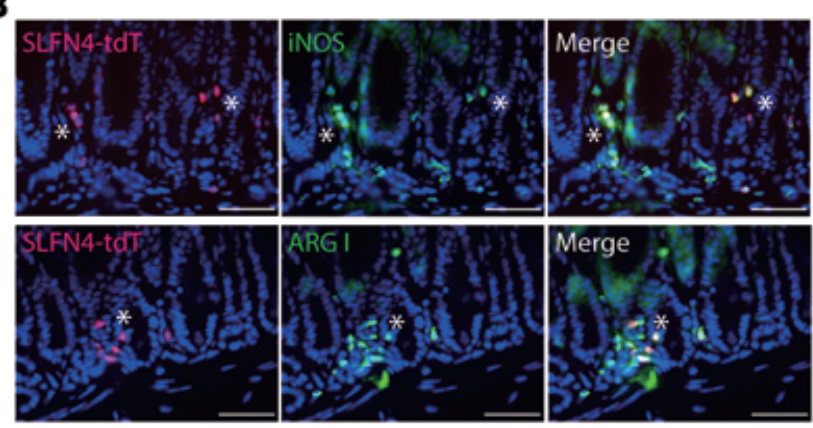

C
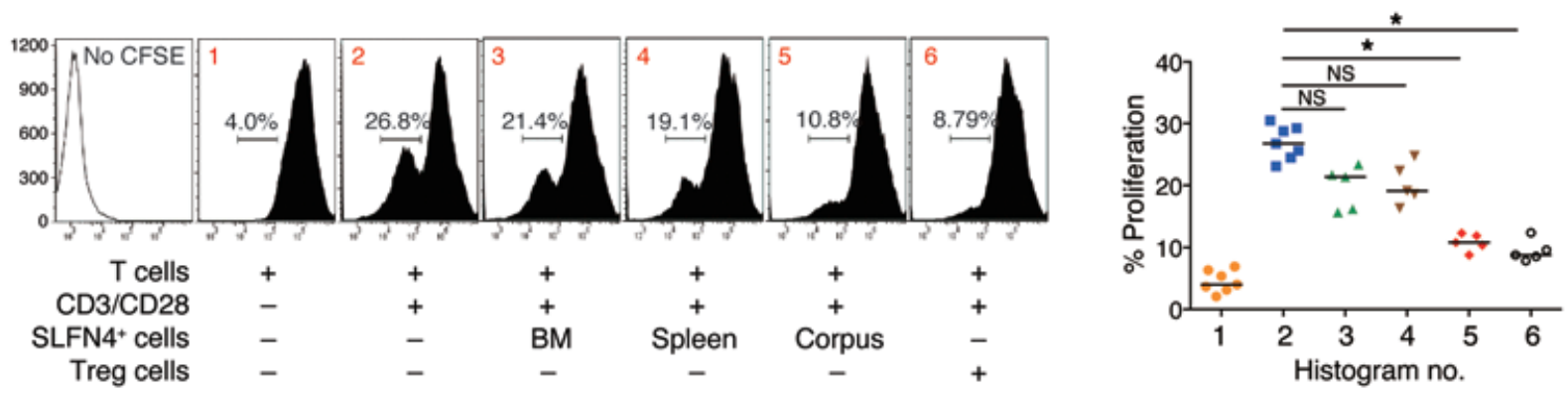

Figure 4. SLFN4 ${ }^{+}$cells from gastric corpus suppress T cell proliferation. (A) SLFN4 ${ }^{+}$cells from bone marrow, spleen, and corpus were analyzed for Ly6G, Ly6C, and iNOS expression. Gated SLFN4+iNOS+ cells from the corpus were further analyzed for arginase I (ARG1). Median percentage (interquartile range) for each gated subpopulation shown in red for $n=4$ mice per tissue. (B) Representative images from $n=4$ mouse stomachs of SLFN4-tdT ${ }^{+}$cells (red) colocalized with ARG1 (green) or iNOS (green). Scale bars: $20 \mu \mathrm{m}$. Asterisks indicate colocalization. (C) SLFN4 ${ }^{+}$cells were sorted from the indicated tissues of 3 pooled 4-month-infected infected $p C M V$-Shh mice and then cocultured with naive splenic T cells prestained with the CFSE dye. T cell proliferation was initiated with anti-CD3/CD28 microbeads cultured with or without SLFN4 ${ }^{+}$cells for 72 hours, and $\mathrm{CFSE}^{+}$T cells were quantified by flow cytometry. The median percentage of proliferating T cells is shown in the representative histograms (nos. 1-6) plotted for $n=5$ experiments in the scatter graph. Tregs $\left(C D 4{ }^{+} \mathrm{CD}_{25}\right)$ from spleen were used as the positive control. Kruskal-Wallis ANOVA with Dunn's test of multiple comparisons was performed. ${ }^{*} P<0.05$.

the stomach, FACS-sorted SLFN4-tdT ${ }^{+}$cells were Giemsa stained and quantified, revealing that granulocytic cells constituted the majority of SLFN4-tdT ${ }^{+}$cells in the stomach (Figure 3I).

SLFN4 marks MDSCs in the gastric mucosa. Ectopic expression of SHH increased the number of cells that were $\mathrm{CD}^{1} \mathrm{~b}^{+}$ Gr-1 ${ }^{+}$SLFN $4^{+} 4$ months after infection compared with WT mice that acquired the same cell population at 6 months (Supplemental Figure 6). Mouse MDSC markers are defined as $\mathrm{CD} 11 \mathrm{~b}^{+} \mathrm{Gr}-1^{+}$ and subdivided into monocytic (Ly6G- ${ }^{-}{ }^{2} 6 \mathrm{C}^{\mathrm{hi}}$ ) and granulocytic $\left(\mathrm{Ly} 6 \mathrm{G}^{+} \mathrm{Ly} 6 \mathrm{C}^{\mathrm{lo}}\right)$ subtypes. Analysis for $\mathrm{Gr}-1$ subunits confirmed that the SLFN4 ${ }^{+}$cells from bone marrow, spleen, and stomach were $\mathrm{Ly} 6 \mathrm{G}^{+} \mathrm{Ly} 6 \mathrm{C}^{\mathrm{lo}}$, consistent with a granulocytic MDSC phenotype (Gr-MDSCs) (Figure 4A). Next, we queried whether the gastric SLFN $4^{+}$population acquired the ability to suppress $\mathrm{T}$ cells. MDSCs suppress $\mathrm{T}$ cells in part by depleting $\mathrm{L}$-arginine from the microenvironment. L-Arginine depletion by MDSCs suppresses CD3- $\zeta$ chain biosynthesis, which subsequently blocks $\mathrm{T}$ cell proliferation during the $G_{0}-G_{1}$ phase of the cell cycle, ultimately reduc- ing T cell cytokine production (20). MDSCs express high levels of inducible NOS (iNOS, or NOS2) and arginase I (ARG1), which consume $\mathrm{L}$-arginine in the microenvironment to produce nitric oxide and ROS (26). We found that the SLFN $4^{+}$cells were positive for iNOS in the corpus but not in the bone marrow or spleen, suggesting that acquisition of the MDSC phenotype occurred in the stomach (Figure 4A). Gated SLFN4 ${ }^{+}$iNOS $^{+}$cells from the corpus were further identified as ARG1 ${ }^{+}$cells. Indeed, further analysis of these cells in the stomach demonstrated colocalization of SLFN4tdT with iNOS and ARG1 (Figure 4B). A CFSE-based T cell suppression assay was performed to detect the suppressive function of $\mathrm{SLFN}^{+}{ }^{+}$cells. When the SLFN $4{ }^{+}$cells from the stomach were sorted and cocultured with activated T cells, we observed a 52\% suppression of T cell activation with the SLFN $4^{+}$cells isolated from the stomach, but only minimal suppression when the SLFN $4^{+}$cells were isolated from the bone marrow or spleen (Figure 4C). Tregs exhibited a $62 \%$ suppression of $\mathrm{T}$ cell activation. Therefore, we concluded that the appearance of SLFN $4^{+}$cells in the stomach was 

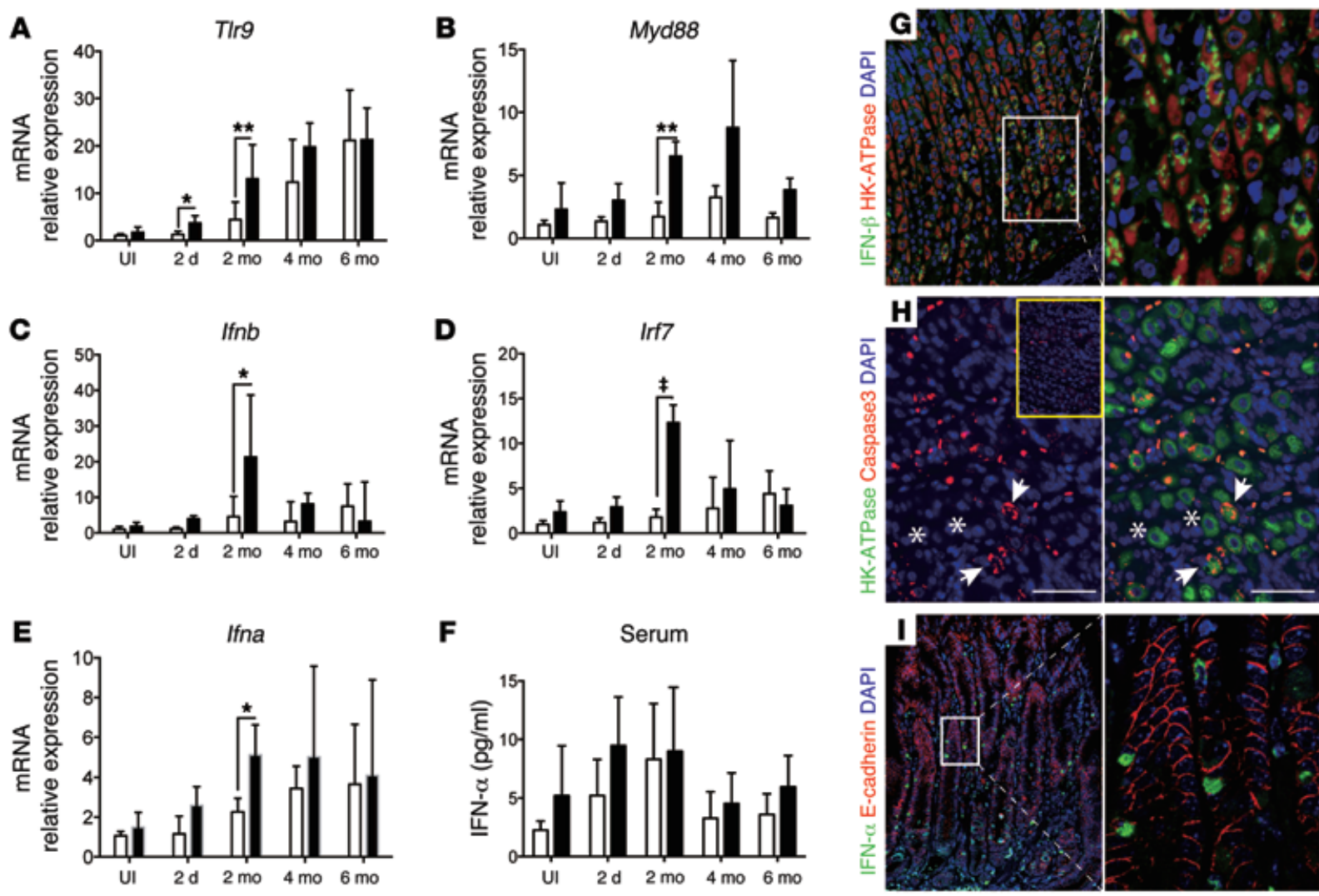

Figure 5. Analysis of DAMP signaling components in $\boldsymbol{H}$. felis-infected corpus. Total RNA was extracted from $\boldsymbol{H}$. felis-infected WT and $p C M V$-Shh corpus lysates and analyzed by RT-qPCR for (A) TIrg, (B) Myd88, (C) Ifnb1, (D) Irf7, and (E) Ifna mRNA at the indicated time points, and then expressed as fold change relative to uninfected WT mice (UI). (F) Serum IFN- $\alpha$ levels determined by ELISA. Shown are the median and interquartile range for $n=8-10$ mice per group over 3 experiments. One-way ANOVA followed by Tukey's multiple comparisons test on log-transformed values was performed. ${ }^{*} P<0.05$, ${ }^{* *} P<0.01,{ }^{\ddagger} P<0.001$. Immunofluorescence images for (G) IFN- $\beta 1$ and (H) cleaved caspase- 3 with $\mathrm{H}^{+}-\mathrm{K}^{+}$-ATPase (HK-ATPase) in the gastric corpus of 2-month $\mathrm{H}$. felis-infected $p C M V$-Shh mice. Inset: Image of uninfected $p C M V$-Shh gastric corpus stained for cleaved caspase-3. Asterisks and arrows indicate nonapoptotic and apoptotic cells, respectively. G: left, original magnification, $\times 200$; G, right, and $\mathbf{H}$ : magnification, $\times 400$ ); (I) Images for IFN- $\alpha$ with E-cadherin in 4-month-infected $p C M V$-Shh mice. I, left: original magnification, $\times 200$; I, right: magnification, $\times 600$. White boxes indicate enlarged regions. Images in $\mathbf{G}-\mathbf{I}$ are representative of $n=3$ mouse stomachs.

a three-step process requiring a pool of circulating SLFN $4^{+}$cells (which was expanded by SHH overexpression), accumulation of these cells in the corpus in response to the Helicobacter infection, and then acquisition of the ability to suppress $T$ cell proliferation.

Type I IFNs regulate SLFN4. Van Zuylen et al. previously showed that type I interferons induce Slfn 4 mRNA expression in bone marrow-derived macrophages through a MYD88-independent mechanism (18). Moreover IFN- $\alpha$ strongly induces all human schlafen family members (27). Since induction of Slfn4 expression correlated with the acquisition of MDSC function coincident with SPEM, we analyzed gastric lysates from infected WT and $p C M V$-Shh mice for type I interferon expression. Previous reports showed that macrophages, dendritic cells, and $\mathrm{CD}^{+}$cells increase gene expression of Toll-like expression receptor $9(\mathrm{Tl} 9)$ in mouse gastric tissue following $H$. pylori infection (28). TLR9 recognizes unmethylated bacterial CpG DNA and induces production of a variety of cytokines, including type I IFNs (IFN- $\alpha / \beta)$ (29). Moreover, damage-associated molecular patterns (DAMPs) from stressed, injured, or dying cells primarily activate TLR9 via MYD88, IRF3, and eventually IFN- $\beta$ (30). Secreted IFN- $\beta$ binds and activates the type I IFN receptor in an autocrine or para- crine manner, leading to IRF7 translocation to the nucleus and expression of IFN- $\alpha$. Thus, we posited that Helicobacter infection activates TLR9 on gastric epithelial cells, triggering events that culminate in the local release of an immune modulator such as IFN- $\alpha$. Indeed, Tlr 9 expression gradually increased over 6 months in both groups of infected mice, whereas Myd88 expression was elevated in $p C M V$-Shh mouse stomachs, with peak expression at 2 and 4 months (Figure 5, A and B). By contrast, Ifnb1 and Irf7 expression in the $p C M V$-Shh mice peaked sharply at 2 months, then returned to near baseline by 4 months; while Ifna mRNA was highly expressed in the infected $p C M V$-Shh mouse stomachs and peaked at 2 months, then remained elevated in both groups (Figure $5, \mathrm{C}-\mathrm{E})$. By comparison, serum IFN- $\alpha$ protein levels trended upward during the first 2 months of the infection, but there was no statistically significant difference in the levels between infected WT and $p C M V$-Shh mice (Figure $5 \mathrm{~F}$ ), suggesting that differential regulation of Ifna correlated with maximal Slfn 4 expression and the histological changes only within infected gastric tissue rather than in response to circulating IFN- $\alpha$ (compare Figure $3 \mathrm{~F}$ with Figure 5, E and F). Immunofluorescence demonstrated that IFN- $\beta$ protein was expressed in $\mathrm{H}^{+}-\mathrm{K}^{+}$-ATPase ${ }^{+}$parietal cells (Figure $5 \mathrm{G}$ ). 
A

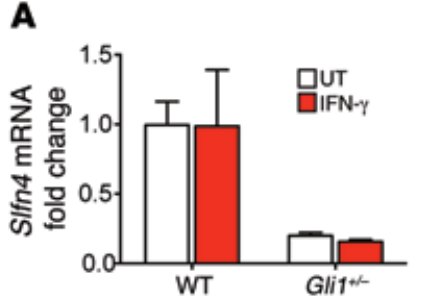

B

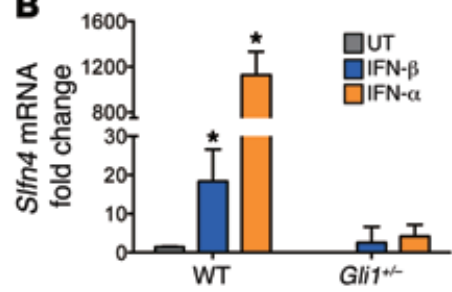

D

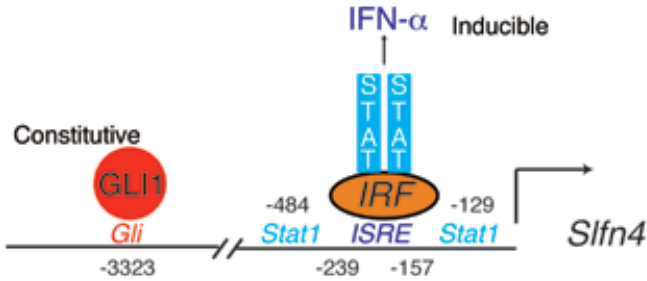

Slfn4

C

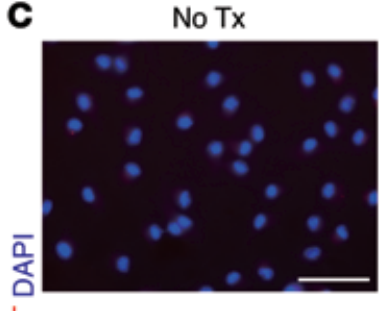

Slfn4-tdT

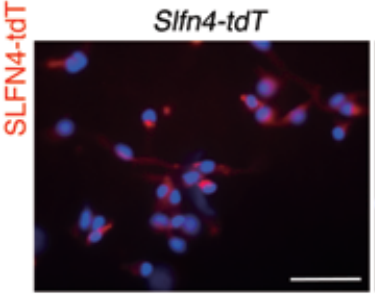

No IFN- $\alpha$

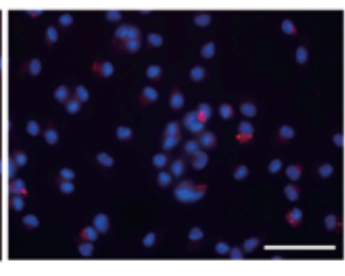

Slfn4-tdT Gli1 $\$-$
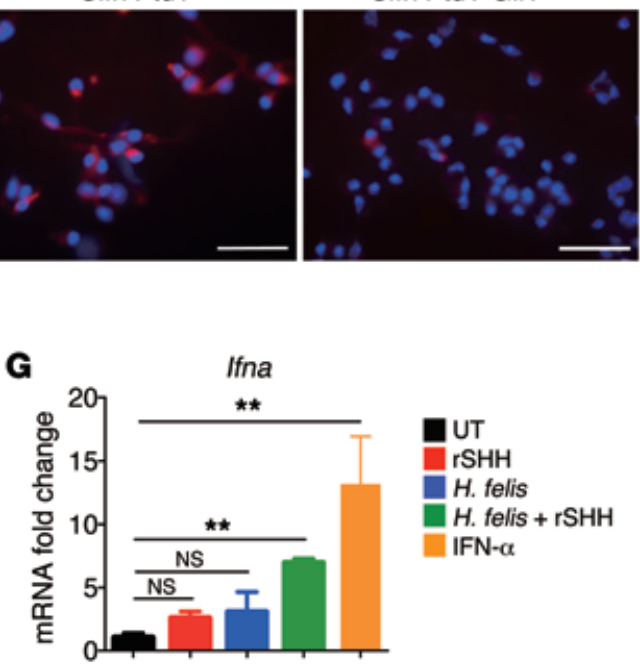

E

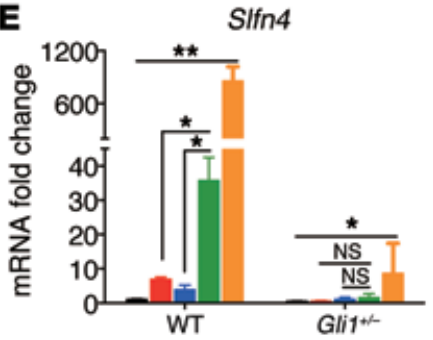

$\mathbf{F}$

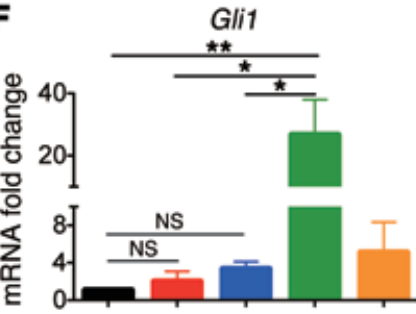

Figure 6. SHH synergizes with $\boldsymbol{H}$. felis to stimulate Gli1 and SIfn4 in primary myeloid cells. (A) Peritoneal myeloid cells from WT or Clit+- mice were treated with IFN- $\gamma(800 \mathrm{U} / \mathrm{ml})$, and SIfn4 mRNA was analyzed by RT-qPCR. (B) SIfn4 mRNA expression determined after treating peritoneal cells with IFN- $\alpha$ (800 $\mathrm{U} / \mathrm{ml}$ ) or IFN- $\beta(200 \mathrm{ng} / \mathrm{ml})$ for 24 hours. (C) Representative image of SLFN4-tdT+ cells visualized after culturing primary myeloid cells from Slfn4-tdT mice treated ex vivo with IFN- $\alpha$ but no Tx, Tx but no IFN- $\alpha$, or Tx with IFN- $\alpha$; or SIfn4-tdT $T^{+}$Gli ${ }^{+1-}$ cells treated with Tx and IFN- $\alpha$. Scale bars: $20 \mu \mathrm{m}$. Replicated with 3 mice per genotype. (D) Schematic of SIfn4 promoter, with GLI1 and IRF binding sites indicated. Primary peritoneal myeloid cells collected from WT or Clit ${ }^{-/-}$ mice were treated with recombinant mouse SHH ligand ( $\mathrm{rSHH}$ ), $100 \mathrm{CFU}$ live $\mathrm{H}$. felis, or both. (E) Slfn4 mRNA in WT and Gli1 ${ }^{-1-}$ cells; (F) Gli1 mRNA or (G) Ifna mRNA in WT primary myeloid cells. Shown for $\mathbf{A}$ and $\mathbf{B}$ and $\mathbf{E}-\mathbf{G}$ are the median and interquartile range for $n=3$ experiments performed in triplicate per genotype. One-way ANOVA followed by Tukey's multiple comparisons test on log-transformed values was performed. ${ }^{*} P<0.05,{ }^{* *} P<0.01$.

Coexpression of $\mathrm{H}^{+}-\mathrm{K}^{+}$-ATPase and cleaved caspase-3 suggested that the dying parietal cells were the likely source of DAMP signals (Figure $5 \mathrm{H}$ ). IFN- $\alpha$ was expressed on both lamina propria cells and E-cadherin ${ }^{+}$cells (Figure $5 \mathrm{I}$ ). Therefore, the source of IFN- $\alpha$ was further analyzed by flow cytometry (Supplemental Figure 7), showing that gated IFN- $\alpha^{+}$cells were a mixture of plasmacytoid dendritic cells with E-cadherin ${ }^{+}$PDCA- $1^{+}$surface markers $(31,32)$ and $\mathrm{E}$-cadherin ${ }^{-} \mathrm{CD} 8 \mathrm{a}^{+}$cells, which are likely cytotoxic $\mathrm{T}$ or dendritic cells. Collectively, the results demonstrated a time-dependent increase in DAMP pathway components after Helicobacter infection, which correlated with Slfn4 expression and the emergence of MDSCs, whereas ectopic SHH production accentuated the extent of the induction.

Type I interferon induction of Slfn 4 and MDSC function is GLII dependent. Since peak Slfn 4 gene expression correlated with SPEM and the acquisition of MDSC function, we further analyzed interferon regulation of Slfn 4 expression in vitro using primary myeloid cells elicited from the peritoneum. Helicobacter infection is typically associated with a Th1 immune response and elevated IFN- $\gamma$ expression $(33,34)$. However, IFN- $\gamma$, a type 2 interferon, did not induce Slfn 4 expression (Figure 6A). Prior studies reported that schlafens are regulated by type I interferons $(18,35)$. Compar- ing Slfn 4 induction by either IFN- $\alpha$ or IFN- $\beta$ revealed that IFN- $\alpha$ was a more potent inducer of Slfn 4 gene expression than IFN- $\beta$ and that both required GLI1 (Figure 6B). To directly confirm that the SLFN $4{ }^{+}$cells responded directly to IFN- $\alpha$, we generated primary myeloid cells from the Slfn4-tdT and Slfn4-eGFP-CreER $R^{T 2}$ Rosa26-LSL-tdTomato Gli1 ${ }^{+/ n L a c Z}$ (referred to here as Slfn4-tdT $\left.\mathrm{Gli1}^{+/}\right)$mouse lines and then treated both populations of primary myeloid cultures with IFN- $\alpha$. No epifluorescence was observed in the absence of Tx/with IFN- $\alpha$ or with Tx/without IFN- $\alpha$ (Figure $6 \mathrm{C}$ ). However, after Tx and IFN- $\alpha$ treatment, epifluorescent SLFN $4{ }^{+}$cells were observed in primary myeloid cells isolated from the Slfn4-tdT but not the Slfn4-tdT Gli1 ${ }^{+/-}$mouse lines (Figure 6C).

The Slfn 4 promoter consists of a Gli binding site at -3323 (16) and several STAT/ISRE elements at -239 near the start site of transcription (18) (Figure 6D). Slfn4 promoter activity required GLI1; however, elevated SHH levels did not appear sufficient to boost Slfn4 expression in the corpus (Figure $3 \mathrm{~F}$ ), whereas peak induction required IFN- $\alpha$, suggesting synergy between the $\mathrm{HH}$ pathway and an inflammatory signal. To test this concept in the context of a Helicobacter infection, we treated peritoneal myeloid cells from WT and $\mathrm{Gli1}^{-1-}$ mice with recombinant $\mathrm{SHH}(\mathrm{rSHH}), \mathrm{H}$. felis, or both $\mathrm{rSHH}$ and $H$. felis. Although $\mathrm{rSHH}$ and $H$. felis treatment was 
A
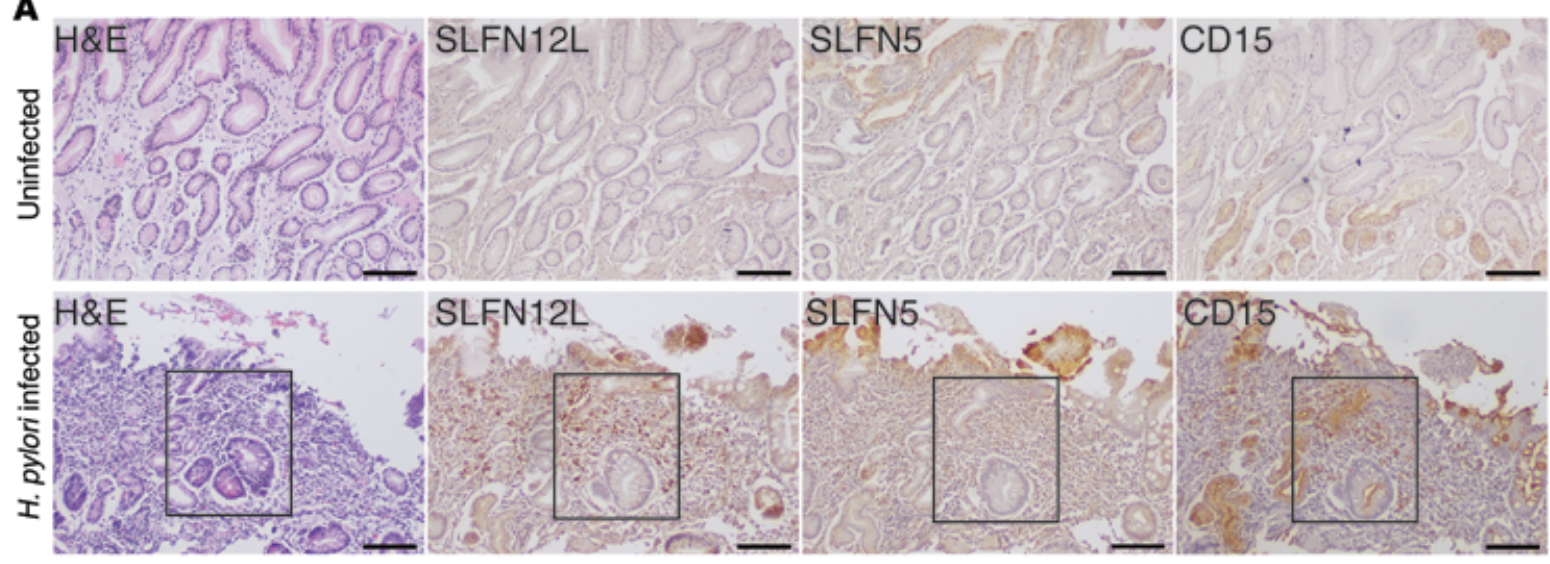

B

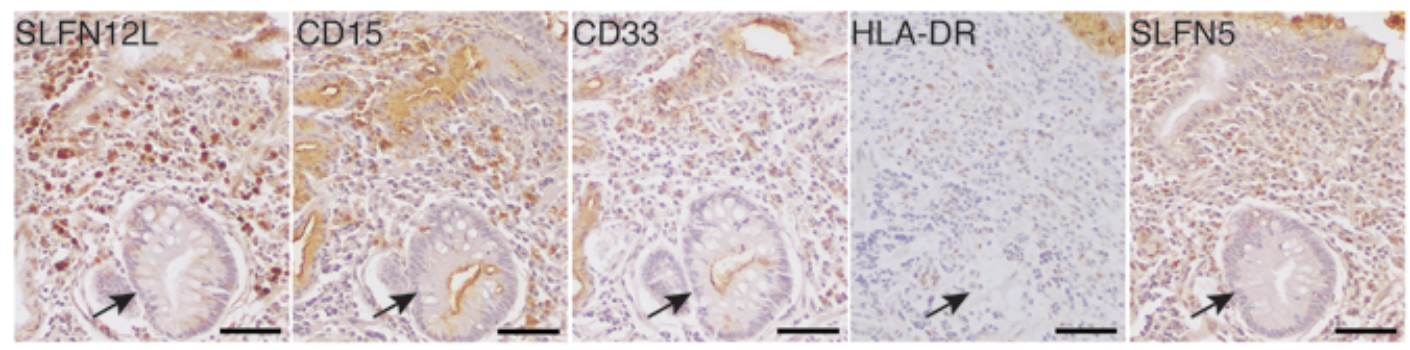

C
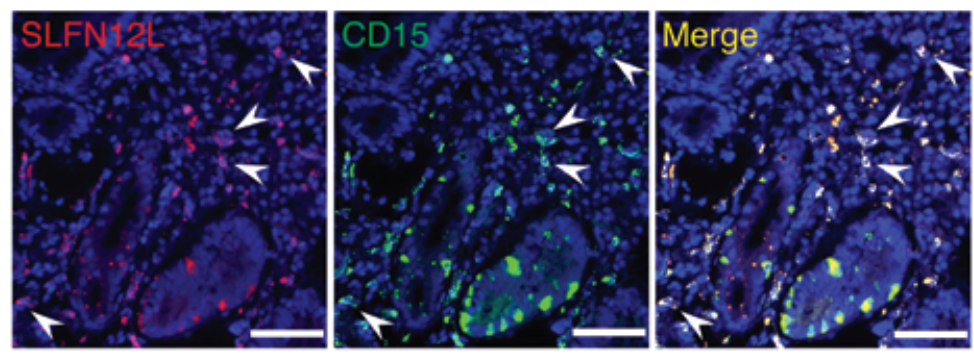

Figure 7. SLFN12L and SLFN5 associated with Gr-MDSCs and intestinal metaplasia in human stomach. (A) Representative images showing H\&E and IHC for SLFN12L, SLFN5, and CD15 in stomach from $n=6$ uninfected and $n=6 \mathrm{H}$. pylori-infected subjects with intestinal metaplasia. Scale bars: $100 \mu \mathrm{m}$. (B) Boxed region expanded in IHC for SLFN12L, CD15, CD33, HLA-DR, and SLFN5. Intestinal metaplastic glands are indicated (arrows). Scale bars: $50 \mu \mathrm{m}$. (C) Immunofluorescence images for SLFN12L (red) and CD15 (green). Arrowheads indicate colocalization. Scale bars: $50 \mu \mathrm{m}$.

sufficient to modestly induce Slfn4 mRNA, synergistic induction of Slfn 4 was observed when the cells were treated with both $\mathrm{HH}$ ligand and $H$. felis (Figure 6E). In addition, IFN- $\alpha$ remained the more potent inducer of Slfn 4 in WT cells, while $95 \%$ of the inducible expression was abolished when the $\mathrm{Gli1}^{-/-}$cells were treated, demonstrating that Gli1 was required for the Slfn 4 promoter to be IFN- $\alpha$ responsive, consistent with synergy between the two pathways. Indeed synergistic induction of Gli1 mRNA was observed with rSHH and $H$. felis, but IFN- $\alpha$ did not strongly induce Gli1 (Figure 6F). By contrast, IFN- $\alpha$ treatment induced Ifna expression in myeloid cells, while rSHH and $H$. felis alone or combined were weak activators of Ifna gene expression (Figure 6G).

Finally, we examined whether IFN- $\alpha$ treatment of primary peripheral myeloid cells was sufficient to induce MDSC surface markers and function. IFN- $\alpha$ induced Slfn 4 expression in $\mathrm{CD}_{11} \mathrm{~b}^{+} \mathrm{Gr}-1^{+}$to a greater extent than $\mathrm{CD} 11 \mathrm{~b}^{+} \mathrm{Gr}-1^{-}$populations (Supplemental Figure 8A). However, only Slfn4 mRNA induction in the double-positive $\mathrm{CD} 11 \mathrm{~b}^{+} \mathrm{GR}-1^{+}$population was Gli1 dependent (Supplemental Figure 8, B and C). Thus, we concluded that IFN- $\alpha$ strongly induced Slfn 4 expression in only a subset of CD11b ${ }^{+} \mathrm{GR}-1^{+}$cells. Gli1-dependent $i$ Nos and Arg1 mRNA expression were highest in the SLFN4 $4^{+}$compared with SLFN4 $4^{-}$cells after IFN- $\alpha$ treatment (Supplemental Figure 9, A and B). Moreover, the ability to suppress $\mathrm{T}$ cell function was greatest only in the CD $11 \mathrm{~b}^{+} \mathrm{Gr}-1^{+} \mathrm{SLFN} 4^{+}$cells compared with the SLFN4 $4^{-}$population (Supplemental Figure 9C).

SLFNs associated with Gr-MDSCs and intestinal metaplasia in human stomach. Since the mouse data suggested that SLFNs might function as potential biomarkers for gastric cancer precursor lesions such as intestinal metaplasia in human subjects, we examined whether SLFNs are expressed in $\mathrm{H}$. pylori-infected individuals and related to MDSCs. Human MDSC markers are defined as CD $33^{+}$ HLA-DR ${ }^{-1 / 0}$, and subdivided into monocytic $\left(\mathrm{CD}^{+} 4^{+}\right)$and granulocytic $\left(\mathrm{CD}^{+} 5^{+}\right)$subtypes. Since SLFN4 does not exist in the human genome, we analyzed SLFN12L as the human ortholog to the mouse Slfn 4 gene based on sequence homology (18) and SLFN5 as the predominant type I IFN-regulated human SLFN gene in human tissues $(27,36)$. Normal gastric mucosa without $H$. pylori infection was neg- 
ative for both SLFN12L- and SLFN5-expressing immune cells, but $\mathrm{SLFN}^{+}$cells were observed in $H$. pylori-infected gastric mucosa with intestinal metaplasia (Figure 7A). These immune cells were also positive for the Gr-MDSC marker CD15 and in each patient were near metaplastic glands in at least 2 fields. Further analysis showed that SLFN12L $\mathrm{L}^{+}$cells are $\mathrm{CD} 15^{+} \mathrm{CD} 33^{+} \mathrm{HLA}-\mathrm{DR}{ }^{\text {lo }}$, consistent with the Gr-MDSC designation (Figure 7, B and C). Therefore, human SLFN12L expression increases in $H$. pylori-infected individuals with intestinal metaplasia and marks a Gr-MDSC subpopulation.

\section{Discussion}

Myeloid cells home from the bone marrow to peripheral organs to fight invading organisms. Infection by Helicobacter induces acute and then chronic gastritis, initiating the development of gastric metaplasia in some individuals and cancer in about $1 \%$ of those infected (1). Yet it is not well understood what component of the chronic inflammatory milieu provides the impetus to propel the mucosa toward cellular atrophy and metaplasia, ultimately presaging the development of cancer. Petersen et al. recently reported that M2 macrophages promote the advancement of SPEM in the presence of inflammation induced by treatment of mice with DMP-777 and L635 (37). However, with chemical induction, oxyntic gland metaplasia develops very rapidly relative to bacterial pathogenesis and may not necessarily recapitulate the events occurring over a prolonged time course as observed in humans. Here we report that canonical $\mathrm{HH}$ signaling plays an essential role in the transition from chronic inflammation to gastric metaplasia (SPEM). Subtyping a population of immune cells responsible for the metaplasia, we found that the GLI1 target gene Slfn 4 marks a subset of CD $11 b^{+}$Gr- $1^{+}$Ly $6 G^{+}$Ly $6 G^{\text {lo }}$ cells, which acquire the ability to suppress $\mathrm{T}$ cell proliferation within the infected stomach. Examining the infected gastric mucosa in 2-month intervals over 6 months revealed that induction of mucosal IFN- $\alpha$ correlated with both SPEM development and the emergence of SLFN4 ${ }^{+}$cells. Recombinant SHH, live Helicobacter, or IFN- $\alpha$ treatment of peritoneal-derived myeloid cell cultures induced Slfn 4 mRNA, providing an ex vivo approach to study the process of MDSC development. The data presented here support a novel pathway for metaplastic induction, where $\mathrm{HH}$ signaling synergizes with regulatory cytokines to create a permissive environment for preneoplastic transformation of the gastric mucosa.

The early phase of gastric cancer pathogenesis, especially with intestinal metaplasia, tends to express high levels of $\mathrm{SHH}$ ligand (38). However, the effect of SHH on gastric tumor initiation and growth has not been well documented. Acute Helicobacter infection in mice increased SHH blood levels (within 2 days), in agreement with a report by Schumacher et al. that secreted SHH diffuses from the stomach through the circulation and is interpreted by the immune system as a help signal to direct circulating immune cells to the infected stomach (14). Once specific myeloid cells express GLI1 in response to SHH ligand, they become primed to respond to accumulating DAMPs and TLR9 signal transduction, which ultimately stimulates the release of IFN- $\alpha$ into the microenvironment, perhaps with the initial intent to quench the inflammatory response initiated by Helicobacter (32).

Since oxyntic glands atrophy during chronic Helicobacter infection (39), stressed parietal cells would typically be the opti- mal candidates to release DAMP signals prior to undergoing apoptosis (30). We found that parietal cells express IFN- $\beta$, a proximal cytokine mediator of DAMP signaling that peaked 2 months after the $H$. felis infection. The time course for mucosal Ifna and Slfn4 mRNA expression was consistent with activation of this regulatory pathway, suggesting that induction of DAMPs and Tlr9 expression occurs 2 months after infection. We speculate that the lag period between chronic inflammation and metaplasia permits the accumulation of a sufficient amount of DAMPs from dying parietal cells that gradually increase Tlr9 and type I interferon expression. Indeed, initiation of DAMP signaling coincided with expression of cleaved caspase- 3 by parietal cells, marking their entry into an apoptotic phase during this 2-month lag period prior to complete atrophy and their replacement by metaplastic mucous cells. Similarly, a recent study reported that $H$. pylori infection in mice gradually induces Tlr 9 expression in the gastric mucosa over 6 months, enhances neutrophil recruitment, and dampens the proinflammatory cytokine response (28).

This cascade of events leads to induction of IFN- $\alpha$ coincident with the emergence of SLFN4-expressing MDSCs and SPEM. Thus, GLI1-dependent Slfn4 expression within myeloid cell subpopulations appears to be a reliable marker of the phenotypic transition, since it requires both the epithelial $\mathrm{HH}$ ligand and immune-modulatory cytokine IFN- $\alpha$ for peak expression. Type I interferons have not been frequently found to play a role in the proinflammatory response observed in a Helicobacter infection (40). Since myeloid cells normally encounter Helicobacter-mediated events only in the infected stomach, peak induction of Slfn 4 appears to occur only when tissue IFN- $\alpha$ levels rise above a certain threshold, and could account for the time lag observed prior to the acquisition of the SLFN4+ MDSC phenotype. This transition period represents an important interventional window, since it is well accepted that once metaplasia segues into dysplasia, medical intervention is unlikely to reverse the histologic progression to frank cancer due to the genetic heterogeneity of many epithelial cancers.

Schlafens in immune cells were recently summarized as being a heterogeneous group of genes that potently suppress cell growth, are strongly induced by type I interferons, and primarily exhibit antiviral and antineoplastic effects $(35,41,42)$. Yet little is understood regarding epithelial remodeling by infiltrating $S L F N$ expressing cells. Although we primarily used this GLI1 gene target to probe the role of $\mathrm{HH}$ signaling in preneoplasia, the current understanding of the function of schlafen family members suggests that they exhibit potent regulatory effects depending on the cell type $(43,44)$. Indeed, the analysis of natural mutations in both mouse and human schlafen loci suggests that they normally depress the cell cycle $(45,46)$. Specifically, the elektra phenotype in mice represents a missense mutation in Slfn2 that allows T cells to remain in a semiactivated state, suggesting a role in mediating T cell quiescence $(46,47)$. We report here that Slfn4 expression marks a GLI1-dependent population of myeloid cells with the ability to suppress $\mathrm{T}$ cell function and phenotypic characteristics of granulocytic MDSCs, consistent with one study showing that gastric cancer patients have elevated levels of MDSCs with a granulocyte-like phenotype (48). Moreover, we showed here that the human homologs SLFN12L and SLFN5 correlated with $H$. pylori 
infection and intestinal metaplasia, and coincided with Gr-MDSC markers in human gastric biopsy samples.

H. felis is not a human pathogen and does not express the virulence factors (i.e., $\operatorname{VacA}$ and the cag pathogenicity island) that presumably contribute to bacterial pathogenesis in human subjects $(49,50)$. Nevertheless, H. felis has routinely been used to accelerate the pathological changes in mice that are normally observed in humans $(51,52)$. Indeed, SLFN4 ${ }^{+}$MDSCs in our mouse model induced by infection with $H$. felis correlated with $\mathrm{SLFN}^{+}$immune cells in $H$. pylori-infected patients with intestinal metaplasia, suggesting direct applicability to human cancer pathogenesis. Therefore, SLFNs might function as biomarkers for preneoplastic transformation of the gastric mucosa, a concept that we have begun to test in human samples (36). However, a recent study showed that gastric IL-22 expression correlated with CXCR2-dependent influx of monocytic MDSCs acutely into the stomach after $H$. pylori infection in mice (53). The difference between that report and our study might be related to the time points they studied (49 days after infection) compared with the extended time line reported here (180 days).

We conclude that Slfn4 is perhaps one of several HH-dependent immune genes expressed at low levels in circulating myeloid cells that becomes primed to respond to a threshold level of specific immune mediators after an acute increase in SHH ligand. In this way, $\mathrm{SLFN}^{+} \mathrm{MDSC}$ s would remain quiescent until they engage regulatory signals (HH ligand and type I IFNs) at the appropriate time to dampen the proinflammatory response in the stomach. Presumably, sustained immune dysregulation creates a microenvironment capable of supporting cancer development.

\section{Methods}

Transgenic mice. Generation of the $p C M V$-Shh transgenic mouse line was described previously, and it was genotyped using the following primer pairs: forward 5'-CTGGCTAACTAGAGAACC; reverse 5'-CTTTGGATTCATAGTAGACC (16). Gli1-nLacZ reporter mice were a gift from Alex Joyner (Memorial Sloan Kettering Cancer Center, New York, New York, USA). The mice contain a knock-in of the cDNA for nuclear $\beta$-galactosidase ( $n L a c Z$ ) into exon 2 of the mouse Gli1 gene, which disrupts expression, creating a null allele. The mice were maintained as heterozygous Gli1 $^{+/ n L a c Z}$ and homozygous Gli1 $^{\text {nLacZ/nLacZ }}$ and are herein referred to as $\mathrm{Gli1}^{+-}$and $\mathrm{Gli1}^{-/}$, respectively. Genotyping was achieved using the following primers for the WT Gli1 locus: forward 5'-TGATTTCCATGACCAGTTTCTG; reverse 5'-AGGTGAGACGACTGCCAAGT; and for the Gli1-nLacZ knock-in mice: reverse 5'TCTGCCAGTTTGAGGGGACGAC. Rosa26-LoxSTOPLox-tdTomato reporter mice were purchased from The Jackson Laboratory (B6.Cg-

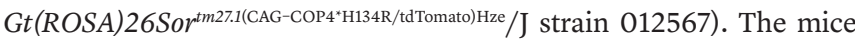
were genotyped using two primer sets: one set for the native ROSA26 locus: ROSA26 locus forward 5'-AAGGGAGCTGCAGTGGAGTA, ROSA26 locus reverse 5'-GGCAAAATCTGTGGGAAGTC; and the second set to detect the Rosa-LSL-Cag-hChR2(H134R)-tdTomatoWPRE targeting vector (tdTomato, or tdT) inserted between exon 1 and exon 2: tdTomato reporter forward 5'-CTGTTCCTGTACGGCATGG, tdTomato reporter reverse 5'-GGCATTAAAGCAGCGTATCC. The $e G F P-C r e E R^{T 2}$ cassette was inserted into exon 5 of the mouse schlafen 4 (Slfn4) gene contained within BAC clone RPCI-23-211B8 (NCBI Clone DB, http://www.ncbi.nlm.nih.gov/clone/666411/) to generate the Slfn4-eGFP-CreER ${ }^{T 2}$ plasmid (JrGang Cheng, University of North Carolina, Chapel Hill BAC Engineering Core, Chapel Hill, North Carolina, USA). The purified Slfn 4 BAC plasmid was microinjected into the pronuclei of fertilized C57BL/ $6 \times$ SJL mouse eggs (University of Michigan Transgenic Core). The presence of the Slfn4$E G F P-C r e E R^{T 2}$ was confirmed using three PCR primer sets: (a) spanning the junction between exon 5 of the Slfn 4 locus and the eGFPCreER $R^{T 2}$ insert cassette, forward 5'-CACCACACACCGTCCAAGTA; reverse $5^{\prime}$-CTTGTAGTTGCCGTCGTCCT using the Bio-Rad thermocycler settings $95^{\circ} \mathrm{C}$ (5 minutes), 10 cycles at $95^{\circ} \mathrm{C}$ ( 1 minute), $60^{\circ} \mathrm{C}$ (1 minute) $\left(-1^{\circ} \mathrm{C}\right.$ per cycle), $72^{\circ} \mathrm{C}$ ( 1 minute), 35 cycles $95^{\circ} \mathrm{C}$ (1 minute), $50^{\circ} \mathrm{C}$ ( 1 minute), $72^{\circ} \mathrm{C}$ ( 1 minute), $72^{\circ} \mathrm{C}$ ( 5 minute), $4^{\circ} \mathrm{C}$ (hold) to produce a 776-bp amplimer; (b) the eGFP sequence using forward 5'-GACGTAAACGGCCACAAGTT; reverse 5'-CCTCCTTGAAGTCGATGCCC primer set, and settings $95^{\circ} \mathrm{C}$ (5 minutes), 10 cycles $95^{\circ} \mathrm{C}$ ( 1 minute), $60^{\circ} \mathrm{C}$ ( 1 minute) $\left(-1^{\circ} \mathrm{C}\right.$ per cycle), $72^{\circ} \mathrm{C}$ ( 1 minute), 25 cycles $95^{\circ} \mathrm{C}(1$ minute), $50^{\circ} \mathrm{C}$ ( 1 minute), $72^{\circ} \mathrm{C}\left(1\right.$ minute), $72^{\circ} \mathrm{C}$ ( 5 minutes), $4^{\circ} \mathrm{C}$ (hold) to produce a 337-bp product; (c) the Cre cDNA using forward $5^{\prime}$-CATGCTTCATCGTCGGTCC; reverse 5'-GATCATCAGCTACACCAGAG primer set and thermocycler settings $95^{\circ} \mathrm{C}\left(5\right.$ minutes), 35 cycles $95^{\circ} \mathrm{C}$ (30 seconds), $58^{\circ} \mathrm{C}$ ( 40 seconds), $72^{\circ} \mathrm{C}$ ( 40 seconds), $72^{\circ} \mathrm{C}$ ( 2 minutes), $4^{\circ} \mathrm{C}$ (hold) to generate a 412 -bp PCR product (54). The PCR products were verified by DNA sequencing. To lineage trace the SLFN4 ${ }^{+}$cells, Slfn 4 eGFP-CreER ${ }^{T 2}$ transgenic mice were bred to the Rosa26-LSL- $t d$ Tomato reporter mice to generate Slfn4-CreER R2 Rosa26-tdTomato (Slfn4-tdT) mice. Slfn4-tdT mice and Gli1 $^{-/}$mice were bred to generate Slfn4-CreER ${ }^{T 2}$ Rosa26-tdTomato Gli1 ${ }^{+/}$(Slfn4-tdT Gli1/-) mice. All mice were cohoused under the same specific pathogen-free conditions and fasted overnight prior to use, with free access to water. All mice were maintained on a C57BL/6 genetic background.

BMT. The recipient mice (WT, $p C M V-S h h, \mathrm{Gli1}^{-/+}$, or $\mathrm{Gl1}^{-/}$) were lethally irradiated using 2 doses of $5 \mathrm{~Gy}$ with a 1-hour interval between doses. Bone marrow cells $\left(2 \times 10^{6}\right)$ collected from hind leg long bones of 8- to 12-week-old donor mice (Slfn4-tdT mice) were injected into the tail vein of the irradiated recipient mice. After a 6-week engraftment period, chimeric mice were infected with $H$. felis. To determine BMT efficiency, $1 \mathrm{ng}$ of DNA from the bone marrow of chimeric mice was analyzed by qPCR for the Rosa26-tdTomato ( $t d T)$ transgene. The amount of $t d T$ DNA detected in the donor mice was set to 1 . WT mice without BMT were negative for the $t d T$ transgene, and the level was set to 0 . The ratio of the chimeric mice $t d T$ DNA versus donor mice $t d T$ DNA is shown in Supplemental Figure 10, indicating approximately $70 \%$ engraftment efficiency. Samples with a score $<0.5$ were excluded from further analysis, since it indicated less than $50 \%$ engraftment efficiency.

H. felis culture and infection. $H$. felis (CS1 strain) was cultured in sterile-filtered Brucella broth (BD) plus 10\% horse serum (Atlanta Biologicals) using the GasPak EZ Campy Container System (BD Biosciences) at $37^{\circ} \mathrm{C}$ with shaking at $100 \mathrm{rpm}$. The cultures were pelleted at $1,700 \mathrm{~g}$ at room temperature and then resuspended in saline. The optical density of $\mathrm{H}$. felis was measured, and the final concentration was adjusted to $10^{9}$ organisms per $\mathrm{ml}$ of saline. Mice were gavaged 3 times over 5 days with $10^{8} \mathrm{H}$. felis CFU per $100 \mu \mathrm{l}$. Control mice were gavaged with $100 \mu \mathrm{l}$ saline alone.

Tissue preparation. Mice were fasted overnight before euthanization. The squamous forestomach was removed prior to bisecting the stomach along the greater and lesser curvatures and then sectioning each half stomach into longitudinal strips. Tissue from the lesser 
and greater curvatures was fixed overnight in $4 \%$ paraformaldehyde and transferred to $70 \%$ ethanol for subsequent paraffin embedding. The remaining corpus tissue was minced for total RNA and protein extraction as well as tissue dissociation in EDTA for flow cytometry.

Flow cytometry. Stomach or small intestine was minced, incubated in $20 \mathrm{ml} \mathrm{Ca}{ }^{+2} / \mathrm{Mg}^{+2}$-free HEPES-buffered saline solution (CMF-HBSS) supplemented with 5\% FBS (Thermo Fisher Scientific) and 2 mM EDTA (51201, Lonza) with orbital shaking at $250 \mathrm{rpm}$ for 20 minutes at $37^{\circ} \mathrm{C}$. The non-dissociated tissue was removed by passing the 20 -ml cell suspension through a 100- $\mu$ m cell strainer by gravity (Fisher Scientific). The tissue collected in the cell strainer was further digested a second time in $20 \mathrm{ml}$ EDTA media (CMF-HBSS/5\% FBS with $2 \mathrm{mM} \mathrm{EDTA}$ ) at $250 \mathrm{rpm}$ for 20 minutes at $37^{\circ} \mathrm{C}$. After a second round of EDTA chelation, the cell suspension was passed through the cell strainer a third time, minced rapidly, and then transferred to $20 \mathrm{ml}$ of collagenase solution $(1.5 \mathrm{mg} /$ $\mathrm{ml}$ type VIII collagenase dissolved in prewarmed CMF-HBSS/5\% FBS with $40 \mu \mathrm{g} / \mathrm{ml}$ DNase I) for digestion at $200 \mathrm{rpm}$ for 10-20 minutes at $37^{\circ} \mathrm{C}$. After passing through the $100-\mu \mathrm{m}$ cell strainer and centrifuging at $400 \mathrm{~g}$ for 5 minutes at $4^{\circ} \mathrm{C}$, the cell pellet was resuspended in PBS containing 5\% BSA. To collect bone marrow cells, the marrow from the femurs was directly flushed with PBS, and red blood cells were lysed in ACK lysis buffer (10-548E, Lonza). Cells were collected at $400 \mathrm{~g}$ and resuspended in $200 \mu \mathrm{l} \mathrm{PBS}$ containing 5\% BSA (Roche). To collect splenocytes and hepatocytes, the spleen and liver were triturated with a 1-ml syringe and flushed through a $70-\mu \mathrm{m}$ cell strainer into a $50-\mathrm{ml}$ conical tube. The strainer was rinsed twice with HBSS to recover all cells. The cells were collected at $300 \mathrm{~g}$ for 10 minutes, and the pellet was resuspended in ACK lysis buffer with gentle swirling for 2 minutes to lyse red blood cells. The lysis was stopped by adding $12 \mathrm{ml}$ of HBSS, centrifuging at $300 \mathrm{~g}$ for 10 minutes, and then resuspending the cells in PBS containing $5 \%$ BSA. Cells $\left(10^{6}\right.$ in a $100-\mu$ total volume) were blocked with purified rat anti-mouse CD16/CD32 Fc block (BD Biosciences) at $4^{\circ} \mathrm{C}$ for 5 minutes, prior to incubation with $0.25 \mu \mathrm{g}$ CD11b-FITC (BioLegend), Gr-1-APC (BioLegend), Ly6G-APCCy7 (BioLegend), Ly6C-Pacific Blue (BioLegend), CD8a-APC (BioLegend), PDCA-1-PE-Cy7 (eBioscience), rabbit anti-E-cadherin (sc-7870, Santa Cruz Biotechnology Inc.), NOS2FITC (Santa Cruz Biotechnology Inc.), rabbit anti-arginase I (sc-20150, Santa Cruz Biotechnology Inc.), IFN- $\alpha$-FITC (Interferon Source). Prior to staining for intracellular proteins, cells were fixed and permeabilized with the Fix and Perm Kit (Thermo Fisher). Prior to initiating the multicolor analysis, we ran the fluorescence minus one (FMO) control to define the spectral overlap. A single-stained control for each fluorochrome was made for compensation for each experiment. A separate set of cells was stained with isotype control antibodies for the negative control. Isotype control antibodies used were PE isotype control (BioLegend, 400608), Pacific Blue isotype control (BioLegend, 400627), $\mathrm{PE}-\mathrm{Cy} 7$ isotype control (BioLegend, 400618), FITC isotype control (eBioscience, 11-4614-80), APC isotype control (eBioscience, 11-432141), APC-Cy7 (BioLegend, 400623). Cell viability was determined with propidium iodide (Abcam, ab14083). Fluorescent-conjugated secondary antibodies goat anti-rabbit IgG APC conjugate (A-10931) and goat anti-rabbit IgG Alexa Fluor 555 conjugate (A27017) were purchased from Life Technologies. The cells were analyzed using an iCyt Synergy SY3200 flow sorter (Sony Biotechnology Inc.).

Histological analysis. Five-micrometer sections were prepared, deparaffinized, and then rehydrated. Each slide contained sections of well-oriented gastric glandular mucosa, which extended along the greater curvature of the stomach. Slides were stained with H\&E to evaluate the degree of PMN accumulation and metaplasia in the corpus. A veterinary pathologist blinded to the experimental conditions used a semiquantitative scale from 0 to 3 on $\times 200$ histologic fields to score gastric lesions. The absence of inflammatory aggregates, atrophy, or metaplasia was given a score of 0 . For PMNs, more than 3 occasional clusters (3 or more contiguous cells) was scored 1, widespread PMN aggregates at the base of glands were scored 2 , and aggregates throughout the full mucosal thickness were scored 3. For mucous cell metaplasia (loss of parietal cells with replacement by mucous-type cells), the presence of "foamy" epithelial cells in 1-2 glands was scored 1, foamy cells in most fields but less than the entire field was scored 2, and the entire glandular epithelium replaced by foamy mucous cells was scored 3 . The weighted score $=[($ number of fields with a score $=1)$ $+(2 \times$ number of fields with a score $=2)+(3 \times$ number of fields with a score $=3$ ) $/($ total number of fields $\times 3$ ). For each slide, around $12-20$ microscopic fields were scored.

Specific protein expression was identified by immunostaining. Antigen retrieval was performed using $10 \mathrm{mM}$ citric acid buffer $(\mathrm{pH}$ 6). Slides were washed in $0.01 \%$ Triton X-100 in PBS twice, incubated with the serum of the animal in which the secondary antibody was raised for 1 hour. For immunofluorescence staining, slides were incubated with the following antibodies overnight: GSII-FITC (1:1,000, FL-1211, Vector Laboratories), arginase I (1:100, sc-20150, Santa Cruz Biotechnology Inc.), IFN- $\beta$ (1:100, AP30401PU-N, Acris Antibodies), IFN- $\alpha$-FITC (1:200, 22100-3, Interferon Source), $\mathrm{H}^{+}-\mathrm{K}^{+}$-ATPase- $\beta$ (1:1,000, D032-3, Medical and Biological Laboratories), caspase-3 (1:400, 9661, Cell Signaling Technology), clusterin (1:2,000, sc-6420, Santa Cruz Biotechnology Inc.), E-cadherin (1:1,000, sc-7870, Santa Cruz Biotechnology Inc.), CD15 (1:7,500, 13-0159-1631, eBioscience), SLFN12L (1:100, NBP1-91060, Novus). Alexa Fluor-conjugated secondary antibodies (Molecular Probes, Invitrogen) were used to detect primary antibodies at a dilution of 1:500 and then mounted with ProLong Gold Antifade reagent with DAPI (Life Technologies). Species-matched secondary antibodies without primary antibodies were used as the negative controls. Immunofluorescence was visualized using a Nikon Eclipse E800 microscope or Nikon A-1 confocal microscope with NIS acquisition software. For light microscopy, the Vectastain ABC kit (Vector Laboratories) and DAB substrate kit (ab64238, Abcam) were used. Primary antibodies used for DAB staining were SLFN12L (1:100, NBP1-91060, Novus), CD15 (1:7,500, 13-0159-1631, eBioscience), HLA-DR (1:50, 340688, BD), CD33 (1:300, 303414, BioLegend), SLFN5 (1:500, ab121537, Abcam). Secondary antibodies used were goat anti-rabbit (1:1,000, 656140, Invitrogen) and donkey anti-mouse (1:500, BA9200, Vector Laboratories).

$T$ cell suppression assay. $\mathrm{T}$ cells from the mouse spleen were isolated using the EasySep Mouse T Cell Isolation Kit (StemCell Technologies) in which magnetically labeled cells to be excluded are retained by the magnetic field, while unlabeled $\mathrm{T}$ cells pass through and were collected for CFSE labeling using the CellTrace CFSE Cell Proliferation Kit (C34554; Molecular Probes). Purified T cells were cultured in RPMI supplemented with $10 \% \mathrm{FBS}, 2 \mathrm{mM}$ L-glutamine, $1 \mathrm{mM}$ sodium pyruvate, $100 \mathrm{mM}$ nonessential amino acids, $5 \mathrm{mM}$ HEPES free acid, $10 \mathrm{ml}$ of $0.05 \mu \mathrm{M} \beta$-mercaptoethanol, and $100 \mathrm{U} / \mathrm{ml}$ penicillin/streptomycin with $10 \%$ heat-inactivated FBS. To stimulate proliferation, $\mathrm{T}$ cells were cultured with anti-CD3/CD28-coated sulfate latex beads in the medium. Suppression of $\mathrm{T}$ cell proliferation was assayed after the 
addition of SLFN $4^{+}$(tdT, red) cells for 3 days at a T cell/SLFN $4^{+}$cell ratio of 10:1. SLFN $4^{+}$cells were sorted from $H$. felis-infected mouse stomach, bone marrow, or spleen. To ensure that the EDTA-collagenase isolation procedures used to isolate the gastric SLFN4 ${ }^{+}(\mathrm{tdT}$, red) cells did not differentially induce the gastric cell suppressor function relative to the $\mathrm{SLFN}^{+}$(tdT, red) cells, bone marrow cells and splenocytes were prepared in parallel with the gastric tissue. In brief, in order to isolate cells from the bone marrow and spleens to be used in the $\mathrm{T}$ cell suppression assay, the bone marrow was flushed from the long bones with the HBSS/FBS EDTA media (as described above), and spleens were triturated and passed the cell strainer into the EDTA media, with shaking for 20 minutes. The chelation step was repeated. The bone marrow and spleen cells were then incubated in the HBSS/ FBS collagenase/DNAse solution for 20 minutes at $37^{\circ} \mathrm{C}$ with agitation as described above in detail for the minced gastric tissue. The CFSE content in T cells was analyzed by flow cytometry in which low CFSE labeling indicated more proliferation and high-intensity CFSE indicated less proliferation. $\mathrm{T}$ lymphocytes were cultured with $\left(\mathrm{CD} 4^{+} \mathrm{CD} 25^{+}\right)$ splenic Tregs at the same 10:1 ratio were used as the positive control for $\mathrm{T}$ cell suppressor activity.

Thioglycollate-elicited peritoneal cells. Mice were inoculated intraperitoneally with $1 \mathrm{ml}$ of $3 \%$ thioglycollate 2 days prior to isolation of peritoneal myeloid cells. The harvested peritoneal cells were plated onto 6-well plates and allowed to adhere overnight to the plastic. The nonadherent cells were removed by aspiration. Flow cytometry was used to determine that most of the adherent peritoneal cells were positive for CD11b and therefore were designated primary myeloid cells. These cells were treated with $800 \mathrm{U} / \mathrm{ml} \mathrm{IFN}-\gamma, 800 \mathrm{U} / \mathrm{ml} \mathrm{IFN-} \alpha, 200$ $\mathrm{ng} / \mathrm{ml}$ IFN- $\beta, 200 \mathrm{ng} / \mathrm{ml}$ recombinant mouse SHH (rmSHH, R\&D Systems), or $10^{7} \mathrm{CFU}$ of $H$. felis per well ( $10 \mathrm{CFU}: 1$ myeloid cell) for 12 hours prior to analysis. Primary myeloid cells prepared from the Slfn4$t d T$ mice were cultured in DMEM media with $10 \% \mathrm{FBS}$ at $37^{\circ} \mathrm{C}$ and treated with $100 \mathrm{nM} \mathrm{Tx}$ (dissolved in DMSO) for 24 hours to induce Cre recombinase activity ex vivo.

Real-time quantitative PCR. Total RNA from the corpus was extracted from freshly collected tissue in TRIzol (Invitrogen). The RNeasy Mini Kit (QIAGEN) was used for purification of total RNA. RNA $(1 \mu \mathrm{g})$ was DNAse treated (Promega) and used for complementary DNA synthesis using iScript reverse transcriptase (BioRad). RT-qPCR was performed using Platinum Taq DNA polymerase (Invitrogen) on a CFX96 real-time PCR detection system (Bio-Rad), using the following primer sequences $\left(\mathrm{Tm}=65^{\circ} \mathrm{C}\right.$ for all primers): arginase I forward 5'-AACACGGCAGTGGCTTTAACC, reverse 5'-GGTTTTCATGTGGCGCATTC; Gli1 forward 5'-TTGGGATGAAGAAGCAGTTG, reverse 5'-GGAGACAGCATGGCTCACTA; Hprt forward 5'-AGGACCTCTCGAAGTGTTGGATAC, reverse 5'-AACTTGCGCTCATCTTAGGCTTTG; Ifna forward 5'-CGCATCAAAGGACTCATCTGCTG，reverse 5'-CTGCTGCATCAGACAACCTTGC; Ifnb forward 5'-TTCCTGCTGTGCTTCTCCAC, reverse 5'-GATTCACTACCAGTCCCAGAGTC; Ifng forward 5'-TCAAGTGGCATAGATGTGGAAGAA, reverse 5'-TGGCTCTGCAGGATTTTCATG; $i$ Nos forward 5'-CGAAACGCTTCACTTCCAA, reverse 5'-TGAGCCTATATTGCTGTGGCT; Irf7 forward 5'-ACAGGGCGTTTTATCTTGCG, reverse 5'-TCCAAGCTCCCGGCTAAG; Myd88 forward 5'-CAAAAGTGGGGTGCCTTTGC, reverse 5'-AAATCCACAGTGCCCCCAGA; Slfn4 forward 5'-GCCCTCTGTTCAAGTCAAGTGTCC, reverse 5'-CCCAGATGAAATCCTTTCCAC-
GA; clusterin forward 5'-CTGTCCACTCAAGGGAGTAGG; reverse 5'-GTGTCCTCCAGAGCATCCTC; Tff2 forward 5'-CTTGGCCCTGACCTGTATGT, reverse 5'-TAGGTTGCTCAGGTGTCACG; Gif forward: 5'-CССTCTACСTCCTAAGTGTTCTC; reverse 5'-CTGAGTCAGTCACCGAGTTCT.

Western blot analysis. Tissue from the corpus was homogenized in RIPA buffer (Pierce). Western blotting was performed with an SDSPAGE Electrophoresis System (Invitrogen). Briefly, $30 \mu \mathrm{g}$ protein was resuspended in sample buffer, and then electrophoresed on a Novex $4 \%$ to $20 \%$ Tris-Glycine gradient gel (Invitrogen) with Tris running buffer; blotted to PVDF membrane using the iBlot Dry Blotting System (Invitrogen) according to the manufacturer's instructions. The membranes were incubated in nonfat dry milk for 1 hour at room temperature and then sequentially probed with primary antibodies against RFP (600901-379S, Rockland), SLFN4 (sc-8903, Santa Cruz Biotechnology Inc.), or GAPDH (MA5-15738, Thermo Fisher) proteins overnight at $4^{\circ} \mathrm{C}$. Membranes were washed with TBS containing $0.1 \%$ Tween, incubated with IRDye 680-conjugated antibodies (LI-COR), and visualized on an Odyssey infrared imager (LI-COR).

Human gastric tissue analysis. Archived, de-identified tissue sections of endoscopic biopsies from 6 urease-positive (Campylobacter-like organism [CLO] test) and 6 urease-negative subjects were analyzed for human MDSC markers CD15 (LewisX), CD33, and HLADR and schlafen proteins. Intestinal metaplasia was documented on the H\&E-stained sections by Joel Greenson, Gastrointestinal Pathology, University of Michigan. Dual-positive CD15 and SLFN12L cells were identified in at least 2 fields with intestinal metaplasia at $\times 200$.

Statistics. For all ELISA and qPCR experiments, statistical analysis for significance was performed on the log-transformed values using 1-way ANOVA with Tukey's post hoc test for multiple comparisons. Cell subpopulation computed as percentages and the weighted scores used in the morphometric analysis were analyzed using Kruskal-Wallis 1-way ANOVA with Dunn's test for multiple comparisons (GraphPad Prism). All data were expressed as the median with the interquartile range. $P$ values less than 0.05 were considered statistically significant. The number of mice per group and replicate experiments are indicated in the figure legends.

Study approval. Animal use was approved by the University of Michigan Institutional Animal Care and Use Committee. The human tissue samples used in this study were deidentified archived specimens that do not require IRB regulation, as reviewed by the University of Michigan Medical School Institutional Review Board (IRBMED; ID: HUM00108090).

\section{Author contributions}

LD designed and conducted the experiments, analyzed the results, and drafted and edited the manuscript. MMH, KAE, QL, and ROR acquired data. AP performed immunohistochemical analysis. JLM designed the experiments, analyzed results, and wrote the manuscript.

\section{Acknowledgments}

The authors thank the University of Michigan Transgenic Rodent Core supported by the University of Michigan P30 DK Digestive Disease Center (5P3ODK034933) and acknowledge support from PHS grant P01 DK62041 (to J.L. Merchant). The authors thank Jessy Deshane, Asma Nusrat, and Ben Allen for their careful 
review of the manuscript; JrGang Cheng for creating the Slfn4$e G F P-C r e E R^{T 2}$ construct by BAC recombineering; and University of Michigan statisticians Kerby Shedden, Chris Andrews, and Joel Greenson (University of Michigan Gastrointestinal Pathology) for histopathologic analysis of archived human biopsies.
Address correspondence to: Juanita L. Merchant, Departments of Internal Medicine-Gastroenterology and Molecular and Integrative Physiology, University of Michigan, 109 Zina Pitcher Pl., BSRB 2051, Ann Arbor, Michigan 48109-2200, USA. Phone: 734.936.6365; E-mail:merchanj@umich.edu.
1. Haenszel W, Correa P. Developments in the epidemiology of stomach cancer over the past decade. Cancer Res. 1975;35(11 pt 2):3452-3459.

2. Correa P. Gastric cancer: overview. Gastroenterol Clin North Am. 2013;42(2):211-217.

3. Fox JG, Wang TC. Inflammation, atrophy, and gastric cancer. J Clin Invest. 2007;117(1):60-69.

4. Cancer Genome Atlas Research Network. Comprehensive molecular characterization of gastric adenocarcinoma. Nature. 2014;513(7517):202-209.

5. El-Omar EM, et al. Interleukin-1 polymorphisms associated with increased risk of gastric cancer. Nature. 2000;404(6776):398-402.

6. Tu S, et al. Overexpression of interleukin-1beta induces gastric inflammation and cancer and mobilizes myeloid-derived suppressor cells in mice. Cancer Cell. 2008;14(5):408-419.

7. Blaser MJ, Parsonnet J. Parasitism by the "slow" bacterium Helicobacter pylori leads to altered gastric homeostasis and neoplasia. JClin Invest. 1994;94(1):4-8.

8. González CA, Sanz-Anquela JM, Gisbert JP, Correa P. Utility of subtyping intestinal metaplasia as marker of gastric cancer risk. A review of the evidence. Int J Cancer. 2013;133(5):1023-1032.

9. Schmidt $\mathrm{PH}$, et al. Identification of a metaplastic cell lineage associated with human gastric adenocarcinoma. Lab Invest. 1999;79(6):639-646.

10. Yamaguchi H, Goldenring JR, Kaminishi M, Lee JR. Identification of spasmolytic polypeptide expressing metaplasia (SPEM) in remnant gastric cancer and surveillance postgastrectomy biopsies. Dig Dis Sci. 2002;47(3):573-578.

11. Goldenring JR, Nam KT, Wang TC, Mills JC, Wright NA. Spasmolytic polypeptide-expressing metaplasia and intestinal metaplasia: time for reevaluation of metaplasias and the origins of gastric cancer. Gastroenterology. 2010;138(7):2207-2210.

12. Yeh JM, Hur C, Ward Z, Schrag D, Goldie SJ. Gastric adenocarcinoma screening and prevention in the era of new biomarker and endoscopic technologies: a cost-effectiveness analysis. Gut. 2016;65(4):563-574.

13. Merchant JL. Hedgehog signalling in gut development, physiology and cancer. J Physiol. 2012;590(3):421-432.

14. Schumacher MA, et al. Gastric Sonic Hedgehog acts as a macrophage chemoattractant during the immune response to Helicobacter pylori. Gastroenterology. 2012;142(5):1150-1159.e6.

15 . Waghray $M$, et al. Interleukin- $1 \beta$ promotes gastric atrophy through suppression of Sonic Hedgehog. Gastroenterology. 2010;138(2):562-572.

16. El-Zaatari M, et al. Gli1 deletion prevents helicobacter-induced gastric metaplasia and expansion of myeloid cell subsets. PLoS One. 2013;8(3):e58935.

17. Kolterud A, et al. Paracrine Hedgehog signaling in stomach and intestine: new roles for hedgehog in gastrointestinal patterning. Gastroenterology. 2009;137(2):618-628.

18. van Zuylen WJ, et al. Macrophage activation and differentiation signals regulate schlafen-4 gene expression: evidence for Schlafen- 4 as a modulator of myelopoiesis. PLoS One. 2011;6(1):e15723.

19. Sade-Feldman M, Kanterman J, Ish-Shalom E, Elnekave M, Horwitz E, Baniyash M. Tumor necrosis factor- $\alpha$ blocks differentiation and enhances suppressive activity of immature myeloid cells during chronic inflammation. Immunity. 2013;38(3):541-554.

20. Gabrilovich DI, Nagaraj S. Myeloid-derived suppressor cells as regulators of the immune system. Nat Rev Immunol. 2009;9(3):162-174.

21. Gabrilovich DI, Ostrand-Rosenberg S, Bronte V. Coordinated regulation of myeloid cells by tumours. Nat Rev Immunol. 2012;12(4):253-268.

22. Saqui-Salces M, et al. Inflammation and Gli2 suppress gastrin gene expression in a murine model of antral hyperplasia. PLoS One. 2012;7(10):e48039.

23. Goldenring JR, Nam KT, Mills JC. The origin of pre-neoplastic metaplasia in the stomach: chief cells emerge from the Mist. Exp Cell Res. 2011;317(19):2759-2764.

24. Nam KT, et al. Mature chief cells are cryptic progenitors for metaplasia in the stomach. Gastroenterology. 2010;139(6):2028-2037.e9.

25. McClelland JF. Perspective: photoacoustic spectroscopy: "Linearization and signal recovery in photoacoustic infrared spectroscopy" [Rev. Sci. Instrum. 78, 051301 (2007)]. Rev Sci Instrum. 2007;78(5):050901.

26. Köstlin N, et al. Granulocytic myeloid derived suppressor cells expand in human pregnancy and modulate T-cell responses. Eur J Immunol. 2014;44(9):2582-2591.

27. Katsoulidis E, et al. Role of interferon \{alpha\} (IFN\{alpha\})-inducible Schlafen-5 in regulation of anchorage-independent growth and invasion of malignant melanoma cells. J Biol Chem. 2010;285(51):40333-40341.

28. Otani K, et al. Toll-like receptor 9 signaling has anti-inflammatory effects on the early phase of Helicobacter pylori-induced gastritis. Biochem Biophys Res Commun. 2012;426(3):342-349.

29. Radovic-Moreno AF, et al. Immunomodulatory spherical nucleic acids. Proc Natl Acad Sci U S A. 2015;112(13):3892-3897.

30. Hou W, et al. Strange attractors: DAMPs and autophagy link tumor cell death and immunity. Cell Death Dis. 2013;4:e966.

31. Siddiqui KR, Laffont S, Powrie F. E-cadherin marks a subset of inflammatory dendritic cells that promote $\mathrm{T}$ cell-mediated colitis. Immunity. 2010;32(4):557-567.

32. Van den Bossche J, Malissen B, Mantovani A, De Baetselier P, Van Ginderachter JA. Regulation and function of the E-cadherin/catenin complex in cells of the monocyte-macrophage lineage and DCs. Blood. 2012;119(7):1623-1633.

33. Yamamoto T, Kita M, Ohno T, Iwakura Y, Sekikawa K, Imanishi J. Role of tumor necrosis factor-alpha and interferon-gamma in Helicobacter pylori infection. Microbiol Immunol. 2004;48(9):647-654.

34. Lindholm C, Quiding-Järbrink M, Lönroth H, Hamlet A, Svennerholm AM. Local cytokine response in Helicobacter pylori-infected subjects. Infect Immun. 1998;66(12):5964-5971.

35. Mavrommatis E, Fish EN, Platanias LC. The schlafen family of proteins and their regulation by interferons. J Interferon Cytokine Res. 2013;33(4):206-210.

36. Companioni Nápoles O, et al. SCHLAFEN 5 expression correlates with intestinal metaplasia that progresses to gastric cancer [published online ahead of print March 31, 2016]. J Gastroenterol. doi:10.1007/s00535-016-1202-4.

37. Petersen CP, Weis VG, Nam KT, Sousa JF, Fingleton B, Goldenring JR. Macrophages promote progression of spasmolytic polypeptide-expressing metaplasia after acute loss of parietal cells. Gastroenterology. 2014;146(7):1727-1738.

38. Lee SY, et al. Sonic hedgehog expression in gastric cancer and gastric adenoma. Oncol Rep. 2007;17(5):1051-1055.

39. Neu B, et al. TNF-alpha induces apoptosis of parietal cells. Biochem Pharmacol. 2003;65(10):1755-1760.

40. Mitchell P, et al. Chronic exposure to Helicobacter pylori impairs dendritic cell function and inhibits Th1 development. Infect Immun. 2007;75(2):810-819.

41. Schwarz DA, Katayama CD, Hedrick SM. Schlafen, a new family of growth regulatory genes that affect thymocyte development. Immunity. 1998;9(5):657-668.

42. Abdel-Mohsen M, et al. Select host restriction factors are associated with HIV persistence during antiretroviral therapy. AIDS. 2015;29(4):411-420.

43. Kuang CY, Yang TH, Zhang Y, Zhang L, Wu Q. Schlafen 1 inhibits the proliferation and tube formation of endothelial progenitor cells. PLoS One. 2014;9(10):e109711.

44. Chaturvedi L, Sun K, Walsh MF, Kuhn LA, Basson MD. The P-loop region of Schlafen 3 acts within the cytosol to induce differentiation of human Caco-2 intestinal epithelial cells. Biochim Biophys Acta. 2014;1843(12):3029-3037.

45. Recher M, et al. Genetic variation in schlafen genes in a patient with a recapitulation of the murine Elektra phenotype. JAllergy Clin Immunol.2014;133(5):1462-1465.e5.

46. Berger M, et al. An Slfn2 mutation causes lymphoid and myeloid immunodeficiency due to loss of immune cell quiescence. Nat Immunol. 2010;11(4):335-343.

47. Derbinski J, Schulte A, Kyewski B, Klein L. Pillars 
article: Promiscuous gene expression in medullary thymic epithelial cells mirrors the peripheral self. Nat Immunol. 2001. 2: 1032-1039. J Immunol. 2016;196(7):2915-2922.

48. Wang L, Chang EW, Wong SC, Ong SM, Chong $\mathrm{DQ}$, Ling KL. Increased myeloid-derived suppressor cells in gastric cancer correlate with cancer stage and plasma S100A8/A9 proinflammatory proteins. J Immunol. 2013;190(2):794-804.

49. Arnold IC, et al. Comparative whole genome sequence analysis of the carcinogenic bacterial model pathogen Helicobacter felis. Genome Biol Evol. 2011;3:302-308.

50. Wang MY, Liu XF, Gao XZ. Helicobacter pylori virulence factors in development of gastric carcinoma. Future Microbiol. 2015;10(9):1505-1516.

51. Duckworth CA, Burkitt MD, Williams JM, Parsons BN, Tang JM, Pritchard DM. Murine models of Helicobacter (pylori or felis)-associated gastric cancer. Curr Protoc Pharmacol. 2015;69(14.34):1-35.
52. Sgouras DN, Trang TT, Yamaoka Y. Pathogenesis of Helicobacter pylori infection. Helicobacter. 2015;20(suppl 1):8-16.

53. Zhuang Y, et al. A pro-inflammatory role for Th22 cells in Helicobacter pylori-associated gastritis. Gut. 2015;64(9):1368-1378.

54. Grachtchouk M, et al. Basal cell carcinomas in mice arise from hair follicle stem cells and multiple epithelial progenitor populations. JClin Invest. 2011;121(5):1768-1781. 